\title{
Quadrangular embeddings of complete graphs and the Even Map Color Theorem (with details) ${ }^{1}$
}

\author{
Wenzhong Liu, Serge Lawrencenko, Beifang Chen, M. N. Ellingham ${ }^{4}$ \\ Nora Hartsfield, Hui Yang, Dong Ye ${ }^{8}$ and Xiaoya Zha ${ }^{9}$
}

2 June 2016; revised 19 October 2018 and 22 July 2021

\begin{abstract}
Hartsfield and Ringel constructed orientable quadrangular embeddings of the complete graph $K_{n}$ for $n \equiv 5(\bmod 8)$, and nonorientable ones for $n \geq 9$ and $n \equiv 1(\bmod 4)$. These provide minimal quadrangulations of their underlying surfaces. We extend these results to determine, for every complete graph $K_{n}, n \geq 4$, the minimum genus, both orientable and nonorientable, for the surface in which $K_{n}$ has an embedding with all faces of degree at least 4 , and also for the surface in which $K_{n}$ has an embedding with all faces of even degree. These last embeddings provide sharpness examples for a result of Hutchinson bounding the chromatic number of graphs embedded with all faces of even degree, completing the proof of the Even Map Color Theorem. We also show that if a connected simple graph $G$ has a perfect matching and a cycle then the lexicographic product $G\left[K_{4}\right]$ has orientable and nonorientable quadrangular embeddings; this provides new examples of minimal quadrangulations.
\end{abstract}

Keywords: quadrangular embedding, complete graph, minimal quadrangulation, 4-genus, evenfaced embedding, map coloring, chromatic number.

\footnotetext{
${ }^{1}$ The United States Government is authorized to reproduce and distribute reprints notwithstanding any copyright notation herein.

${ }^{2}$ Department of Mathematics, Nanjing University of Aeronautics and Astronautics, Nanjing 210016, China. Email: wzhliu7502@nuaa.edu.cn.

${ }^{3}$ Russian State University of Tourism and Service, Institute for Tourism and Hospitality, Bldg. 32A, Kronstadt Boulevard, Moscow, 125438, Russia. Email: lawrencenko@hotmail.com.

${ }^{4}$ Hong Kong University of Science and Technology, Clear Water Bay, Kowloon, Hong Kong, China. Email: mabfchen@ust.hk.

${ }^{5}$ Department of Mathematics, Vanderbilt University, Nashville, TN 37240, USA, Email: mark.ellingham@vanderbilt.edu.

${ }^{6}$ Deceased.

${ }^{7}$ Department of Mathematics, Guizhou University, Guiyang, 550025, China. Email: hui-yang@163.com.

${ }^{8}$ Department of Mathematical Sciences, Middle Tennessee State University, Murfreesboro, TN 37132, USA, Email: dong. ye@mtsu . edu.

${ }^{9}$ Department of Mathematical Sciences, Middle Tennessee State University, Murfreesboro, TN 37132, USA, Email: xiaoya.zha@mtsu.edu.
} 


\section{Main results}

In this paper surfaces are connected compact 2-manifolds without boundary. The orientable surface of genus $h$ is denoted $S_{h}$, and the nonorientable surface of genus $k$ is denoted $N_{k}$. The Euler characteristic of a surface $\Sigma$ is denoted $\varepsilon(\Sigma)$, which is $2-2 h$ for $S_{h}$, and $2-k$ for $N_{k}$.

Frequently we want embeddings of a given graph with minimum genus, which have faces that are small, often triangular faces. In particular, the determination of the minimum genus of complete graphs as part of the Map Color Theorem [31] was one of the driving forces behind the development of topological graph theory. However, we can also consider the minimum genus of embeddings with restrictions on face degrees. In this paper we consider embeddings where all faces have degree at least 4, or all faces have even degree. Euler's formula and face/edge counting imply the following.

Observation 1.1. If $\Phi$ is an embedding of an $n$-vertex $m$-edge graph in a surface $\Sigma$ with all faces of degree at least 4 , then $m \leq 2 n-2 \varepsilon(\Sigma)$, with equality if and only if the embedding is cellular and every face degree is 4 . For a complete graph $K_{n}, n \geq 4$, such an embedding has $n(n-5) \leq-4 \varepsilon(\Sigma)$, with equality if and only if the embedding is quadrangular.

In this paper we completely resolve the question of the minimum genus of a surface in which $K_{n}$ has an embedding with all faces of degree at least 4 , or with all faces of even degree. These results also complete the proof of a coloring result, the Even Map Color Theorem. In 1975 Hutchinson [16] showed that the chromatic number bound of the Map Color Theorem can be significantly improved for even-faced embeddings; our results improve her bound in one case and provide sharpness examples. We also provide some constructions for minimal quadrangulations, simple quadrangulations with a minimum number of vertices in a given surface.

Our main results are as follows (see Section 3 for definitions not stated here). For a graph $G$ and positive integer $d$, the orientable d-genus $g_{d}(G)$ and the orientable even-faced genus $g_{\text {even }}(G)$ are the smallest $h \geq 0$ for which $G$ has a cellular embedding in $S_{h}$ with all face degrees at least $d$, or with all face degrees even, respectively. We can similarly define the nonorientable d-genus $\widetilde{g}_{d}(G)$ and the nonorientable even-faced genus $\widetilde{g}_{\text {even }}(G)$ (for convenience we take $\widetilde{g}_{d}(G)$ or $\widetilde{g}_{\text {even }}(G)$ to be 0 if $G$ has a suitable planar embedding).

Theorem 1.2. Let $f(n)=1+\lceil n(n-5) / 8\rceil$. Then

$$
g_{4}\left(K_{n}\right)=f(n) \quad \text { if } n \geq 4, \quad \text { and } \quad g_{\mathrm{even}}\left(K_{n}\right)= \begin{cases}f(n) & \text { if } n \geq 4 \text { and } n \neq 6, \\ f(6)+1=3 & \text { if } n=6 .\end{cases}
$$

For $n=5$, for $n \geq 7$, and for $g_{4}\left(K_{6}\right)$ there is a face-simple closed-2-cell embedding of $K_{n}$ realizing each equation. Such an embedding is quadrangular if and only if $n \equiv 0$ or $5(\bmod 8)$.

Theorem 1.3. Let $\widetilde{f}(n)=2+\lceil n(n-5) / 4\rceil$. Then

$$
\widetilde{g}_{4}\left(K_{n}\right)=\widetilde{g}_{\text {even }}\left(K_{n}\right)= \begin{cases}\widetilde{f}(n) & \text { if } n \geq 4 \text { and } n \neq 5, \\ \widetilde{f}(5)+1=3 & \text { if } n=5 .\end{cases}
$$

For $n=4$ and for $n \geq 6$ there is a closed-2-cell embedding of $K_{n}$ realizing this pair of equations, that is face-simple if $n \geq 6$. Such an embedding is quadrangular if and only if $n \equiv 0$ or $1(\bmod 4)$. 
Let $\chi(\Phi)$ and $\chi^{*}(\Phi)$ denote the number of colors needed to properly vertex-color or face-color, respectively, a graph embedding $\Phi$. We will ignore loops when vertex-coloring and monofacial edges (with the same face on both sides) when face-coloring, so $\chi$ and $\chi^{*}$ are defined for all embeddings.

Theorem 1.4 (Even Map Color Theorem). Let $\Phi$ be a (not necessarily cellular) embedding of a (not necessarily connected) graph (loops and multiple edges allowed) in a surface $\Sigma$. Define

$$
H_{\text {even }}(\Sigma)=\left\lfloor\frac{5+\sqrt{25-16 \varepsilon(\Sigma)}}{2}\right\rfloor \text { if } \Sigma \neq S_{0}, \quad \text { and } c(\Sigma)= \begin{cases}2 & \text { if } \Sigma=S_{0}, \\ H_{\text {even }}(\Sigma)-1 & \text { if } \Sigma=N_{2} \text { or } S_{2}, \\ H_{\text {even }}(\Sigma) & \text { otherwise. }\end{cases}
$$

(a) If every face of $\Phi$ has even degree (individual face boundary components may have odd length), then (ignoring loops when coloring) $\chi(\Phi) \leq c(\Sigma)$.

(b) If every vertex of $\Phi$ has even degree, then (ignoring monofacial edges when coloring) $\chi^{*}(\Phi) \leq$ $c(\Sigma)$.

Moreover, for every surface $\Sigma$ there exist face-simple closed-2-cell embeddings of connected simple graphs, that are quadrangular for (a) and 4-regular for (b), which show that these bounds are sharp.

The following provides new constructions of minimal quadrangulations, as well as giving an alternative proof of some cases of Theorems 1.2 and 1.3.

Theorem 1.5. Let $G$ be a connected simple graph with a perfect matching. Then $G\left[K_{4}\right]$ has a face-simple orientable quadrangular embedding. Moreover, if $G$ also has a cycle, then $G\left[K_{4}\right]$ also has a face-simple nonorientable quadrangular embedding.

Section 2 provides some background to our results, and Section 3 provides precise definitions and preliminary results. Section 4 proves Theorems 1.2 and 1.3, and Section 5 proves the Even Map Color Theorem. Section 6 proves Theorem 1.5, and Section 7 shows that results from Sections 4 and 6 yield minimal quadrangulations. Section 8 contains some final remarks.

This version of this paper contains some details not included in the published version [26].

\section{Background}

The minimum genus of the complete graph $K_{n}$ and conditions for the existence of triangular embeddings of $K_{n}$ were determined as part of the well-known Map Color Theorem [31], which extended the Four Color Theorem to other surfaces. Subsequently there were a number of results showing existence of multiple triangular embeddings of certain complete graphs, such as [1, 24, 34], and then providing lower bounds on the number of nonisomorphic triangular embeddings of $K_{n}$ for certain families of $n$, such as $[2,8,9,20]$.

Less work has been done on quadrangular embeddings of complete graphs, or embeddings of complete graphs with all faces of degree at least 4, or all faces of even degree. By Observation 1.1, $n(n-5)=-4 \varepsilon(\Sigma)$ when there is a quadrangulation of $K_{n}$ in $\Sigma$, which means that $n \equiv 0$ or $5(\bmod 8)$ in the orientable case, and $n \equiv 0$ or $1(\bmod 4)$ in the nonorientable case. Hartsfield 
and Ringel [14, 15] obtained the following results, mostly using current graphs, covering half of the possible values of $n$ for which quadrangular embeddings of $K_{n}$ may exist.

Theorem 2.1 (Hartsfield and Ringel $[14,15])$. A complete graph $K_{n}$ with $n=8$ or $n \equiv 5(\bmod 8)$ has a face-simple orientable quadrangular embedding. A complete graph $K_{n}$ with $n \geq 9$ and $n \equiv 1$ $(\bmod 4)$ has a face-simple nonorientable quadrangular embedding. However, $K_{5}$ has no nonorientable quadrangular embedding.

The embeddings in Theorem 2.1 are minimal quadrangulations. Hartsfield and Ringel also constructed quadrangular embeddings of the generalized octahedron $O_{2 k}=K_{k}\left[\overline{K_{2}}\right]$ that are minimal. We discuss minimal quadrangulations in more detail in Section 7 . The fact that $K_{5}$ has no nonorientable quadrangular embedding was also proved earlier (in dual form) by Hutchinson [16].

Using current graphs, Korzhik and Voss [20] constructed exponentially many nonisomorphic orientable quadrangular embeddings of $K_{8 s+5}$ for $s \geq 1$, and Korzhik [19] constructed superexponentially many nonisomorphic orientable and nonorientable quadrangular embeddings of $K_{8 s+5}$ for $s \geq 2$. Grannell and McCourt [10] constructed many nonisomorphic orientable embeddings of complete graphs $K_{n}$ with faces bounded by $4 k$-cycles for $k \geq 2$, when $n=8 k s+4 k+1$ for $s \geq 1$.

It is natural to ask whether the results in Theorem 2.1 can be extended to the other cases where quadrangular embeddings of $K_{n}$ might exist, namely $n \equiv 0(\bmod 8)$ for orientable embeddings, and $n \equiv 0(\bmod 4)$ for nonorientable embeddings. When $K_{n}$ has a quadrangular embedding it is a minimal quadrangulation, and realizes $g_{4}\left(K_{n}\right)$ and $g_{\text {even }}\left(K_{n}\right)$, or $\widetilde{g}_{4}\left(K_{n}\right)$ and $\widetilde{g}_{\text {even }}\left(K_{n}\right)$. But we can also try to determine these parameters even if $K_{n}$ does not have a quadrangular embedding. Our Theorems 1.2 and 1.3 resolve all of these questions, and we provide new proofs for the existence results in Theorem 2.1.

Some explanation of the origins of this paper is appropriate. In the early 1990s one of us, Hartsfield, developed a technique for constructing quadrangulations by "adding handles using diagonals". She used this technique to derive a number of results on quadrangular embeddings, including that $K_{n}$ has a nonorientable quadrangular embedding when $n \equiv 0(\bmod 4)[12]$. She also applied this to derive results on $\widetilde{g}_{4}\left(K_{n}\right)$ and $\widetilde{g}_{\text {even }}\left(K_{n}\right)$ in a paper that was submitted for publication in 1994 [13]. As indicated in [17], Hartsfield was aware that her results would give sharpness examples for Hutchinson's coloring results [16]. Hartsfield's papers [12, 13] outlined proofs (providing basis cases and examples of inductive steps, such as from $K_{8}$ to $K_{16}$ ) but did not give complete general arguments.

In the late 1990s three of us, Chen, Lawrencenko and Yang (CLY), derived results on $g_{4}\left(K_{n}\right)$ using current graphs [4, 22]. These were submitted for publication in 1998. When Hartsfield and CLY discovered they had been working on similar results, they decided to combine their results into a single paper. Unfortunately, this single paper was never finished. Some researchers were aware of the results of Hartsfield (cited in [17]) and of CLY (cited in [32]) but they were not publicly available.

Around 2015 the remaining four authors, Ellingham, Liu, Ye and Zha (ELYZ), worked on some problems of Craft [6] on quadrangular embeddings of composition graphs. ELYZ realized that their constructions (see Section 6) provided orientable quadrangular embeddings for $K_{n}$ with $n \equiv 0$ $(\bmod 8)$, which did not seem to be in the literature. ELYZ also came up with a diamond sum 
construction (see Section 4) for nonorientable quadrangular embeddings of $K_{n}$ for $n \equiv 0(\bmod 4)$. ELYZ's results were written up [25] and submitted in 2016. After submission of their paper ELYZ were informed of the earlier unpublished results of Hartsfield and CLY. It was decided to combine all of the results into the present joint paper. Although Nora Hartsfield died in 2011 we think it is appropriate to include her as an author.

We hope that eventually the other proofs of Theorems 1.2 and 1.3 using Hartsfield's diagonal technique and current graphs will also appear. For the current graph results, some modification of the index 2 current graphs in $[4,22]$ is required, and we hope to provide nonorientable constructions as well as orientable ones. A paper using a combination of current graphs and Hartsfield's diagonal technique is in preparation [23] and additional papers may follow.

\section{Preliminaries}

\subsection{Graph embeddings}

Our graphs may have loops or multiple edges; simple graphs have neither. We say a graph embedding has some graph property (such as bipartiteness) if the underlying graph has this property. A face of a graph embedding is cellular if it is homeomorphic to an open disk. We often identify a cellular face by referring to its bounding cycle or bounding closed walk. A graph embedding is cellular if every face is cellular, closed-2-cell if it is cellular and every face is bounded by a cycle (with no repeated vertices), and face-simple if every two distinct faces share at most one boundary edge. In this paper all embeddings are cellular unless we specifically refer to a general embedding, which means that faces may have multiple boundary components and internal handles or crosscaps.

Suppose $\Phi$ is a graph embedding in surface $\Sigma$. The degree of a face is the number of sides of edges with which it is incident. A $k$-face is a face of degree $k$, and a $C_{k}$-face is a cellular face bounded by a $k$-cycle. A cellular $k$-face is bounded by a single closed walk of length $k$, which may or may not be a $k$-cycle. The minimum vertex degree and minimum face degree of $\Phi$ are denoted $\delta(\Phi)$ and $\delta^{*}(\Phi)$, respectively. The embedding $\Phi$ is even-vertexed or even-faced if every vertex or

every face, respectively, has even degree. An even-faced noncellular embedding may have individual face boundary component walks of odd length, as long as the overall degree of each face is even. We say $\Phi$ is quadrangular, or a quadrangulation of $\Sigma$, if every face is a $C_{4}$-face. We also refer to a $C_{4}$-face as a quadrilateral. A quadrangulation of $\Sigma$ is minimal if its underlying graph is simple and connected, and there is no quadrangular embedding of a simple graph of smaller order in $\Sigma$. Similarly, a triangulation of a surface $\Sigma$ is an embedding of a graph in $\Sigma$ such that every face is a $C_{3}$-face.

Lemma 3.1 (Euler's inequality). Suppose we have a general embedding of a graph $G$ in a surface of Euler characteristic $\varepsilon$, with $n$ vertices, $m$ edges and $r$ faces. Then $n-m+r \geq \varepsilon$, with equality if and only if the embedding is cellular,

Observation 3.2. Suppose $\Phi$ is a general embedding of a simple graph $G$ and $\delta(\Phi) \geq 2$. Then every face of $G$ of degree at most 5 is bounded by a single cycle. 
Observation 3.3. Suppose $\Phi$ is a general even-faced embedding of a simple connected graph on at least three vertices. Then every face boundary walk has length at least 3 , and hence $\delta^{*}(\Phi) \geq 4$.

Observation 3.4. Suppose $\Phi$ is a quadrangular embedding of a simple connected graph and $\delta(\Phi) \geq$ 3. If $\Phi$ is not face-simple then it contains two faces of the form (uvwx) and (uvxw). Thus, if $\Phi$ is orientable or bipartite then it is face-simple.

\subsection{Graph operations}

Let $G$ and $H$ be simple graphs. The complement of $G$ is denoted $\bar{G}$. The composition (or lexicographic product) of $G$ and $H$, denoted $G[H]$, has vertex set $V(G) \times V(H)$, with two vertices $\left(v_{1}, w_{1}\right)$ and $\left(v_{2}, w_{2}\right)$ adjacent if and only if either (i) $v_{1} v_{2} \in E(G)$ or (ii) $v_{1}=v_{2}$ and $w_{1} w_{2} \in E(H)$. For example, $K_{n}\left[K_{2}\right]$ is the complete graph $K_{2 n}$, and $K_{n}\left[\overline{K_{2}}\right]$ is the generalized octahedron $O_{2 n}=K_{2 n}-n K_{2}$. The join of $G$ and $H$, denoted $G+H$, is the union of $G$ and $H$ together with one edge $u v$ for each $u \in V(G)$ and $v \in V(H)$. For example, $K_{4}+K_{n}$ is the complete graph $K_{n+4}$.

\subsection{The diamond sum}

Let $G$ and $G^{\prime}$ be two simple graphs with embeddings $\Phi$ and $\Phi^{\prime}$ in disjoint surfaces $\Sigma$ and $\Sigma^{\prime}$, respectively. Suppose that $k \geq 1$ and both $G$ and $G^{\prime}$ have a vertex of degree $k$, say $v$ and $v^{\prime}$ respectively. Let $v$ have neighbors $v_{0}, v_{1}, \ldots, v_{k-1}$ in cyclic order around $v$ in $\Phi$, and let $v^{\prime}$ have neighbours $v_{0}^{\prime}, v_{1}^{\prime}, \ldots, v_{k-1}^{\prime}$ in cyclic order around $v^{\prime}$ in $\Phi^{\prime}$. There is a closed disk $D$ that intersects $G$ in $v$ and the edges $v v_{0}, v v_{1}, \ldots, v v_{k-1}$, and so that the boundary of $D$ intersects $G$ at $v_{0}, v_{1}, \ldots, v_{k-1}$. Similarly, there is a closed disk $D^{\prime}$ that intersects $G^{\prime}$ in $v^{\prime}$ and the edges $v^{\prime} v_{0}^{\prime}, v^{\prime} v_{1}^{\prime}, \ldots, v^{\prime} v_{k-1}^{\prime}$ and so that the boundary of $D^{\prime}$ intersects $G^{\prime}$ at $v_{0}^{\prime}, v_{1}^{\prime}, \ldots, v_{k-1}^{\prime}$. Remove the interiors of $D$ and $D^{\prime}$, and identify their boundaries so that $v_{i}$ is identified with $v_{i}^{\prime}$ for $0 \leq i \leq k-1$. The resulting embedding is called a diamond sum of $\Phi$ and $\Phi^{\prime}$ at $v$ and $v^{\prime}$, denoted $\Phi \diamond_{v, v^{\prime}} \Phi^{\prime}$ or just $\Phi \diamond \Phi^{\prime}$. Its graph is denoted $G \diamond G^{\prime}$ and the surface is the connected sum $\Sigma \# \Sigma^{\prime}$. Note that $\Phi \diamond \Phi^{\prime}$ is orientable if and only if both $\Phi$ and $\Phi^{\prime}$ are orientable.

The diamond sum was first used by Bouchet [3] in dual form to derive a new proof of the minimum genus of $K_{m, n}$. Bouchet's construction was later reinterpreted in more general situations in $[18,27,28]$.

The diamond sum of two cellular embeddings is cellular. It is also not difficult to see that if $\Phi$ and $\Phi^{\prime}$ are quadrangular and the diamond sum $\Phi \diamond \Phi^{\prime}$ is simple, then $\Phi \diamond \Phi^{\prime}$ is also quadrangular. To build embeddings in Section 4 that are face-simple and closed-2-cell, we rely on the following technical extension of this observation, which allows $\Phi^{\prime}$ to contain non- $C_{4}$-faces.

Lemma 3.5. Suppose $\Phi$ is a face-simple quadrangular embedding of a simple graph $G, \delta(\Phi) \geq 3$, $v \in V(G)$, and the neighbors of $v$ in $G$ are independent. Suppose $\Phi^{\prime}$ is a closed-2-cell embedding of a simple graph $G^{\prime}, v^{\prime} \in V\left(G^{\prime}\right)$, and every pair of distinct faces of $\Phi^{\prime}$ shares at most one edge of $G^{\prime}-v^{\prime}$. Then $\Phi^{\prime \prime}=\Phi \diamond_{v, v^{\prime}} \Phi^{\prime}$ is a face-simple closed-2-cell embedding and there is a degree-preserving bijection between the non- $C_{4}$-faces in $\Phi^{\prime}$ and the non- $C_{4}$-faces in $\Phi^{\prime \prime}$.

Note that the condition on pairs of distinct faces of $\Phi^{\prime}$ holds if $\Phi^{\prime}$ is face-simple. 
Proof. There are three types of faces in $\Phi^{\prime \prime}$ : (1) those that use only edges of $G-v ;(2)$ those that use edges of both $G-v$ and $G^{\prime}-v^{\prime}$; and (3) those that use only edges of $G^{\prime}-v^{\prime}$. Represent the faces of $\Phi$ using $v$ as $Z_{i}=\left(v v_{i} w_{i} v_{i+1}\right)$ and the faces of $\Phi^{\prime}$ using $v^{\prime}$ as $Z_{i}^{\prime}=\left(v^{\prime} v_{i}^{\prime} \ldots v_{i+1}^{\prime}\right)$, for $0 \leq i \leq k-1$, taking subscripts modulo $k$. Since the neighbors of $v$ are independent, $w_{i}$ is not a neighbor of $v$.

All faces in $\Phi$ and $\Phi^{\prime}$ are bounded by cycles since $\Phi$ and $\Phi^{\prime}$ are closed-2-cell, which implies that faces of type (1) and (3) are bounded by cycles, and all $Z_{i}$ and $Z_{i}^{\prime}$ are cycles. Thus, every face of type (2) is a face $Z_{i}^{\prime \prime}$ obtained by combining paths $Z_{i}-v=v_{i} w_{i} v_{i+1}$ and $Z_{i}^{\prime}-v^{\prime}$ by identifying $v_{i}$ with $v_{i}^{\prime}$ and $v_{i+1}$ with $v_{i+1}^{\prime}$. Since $w_{i}$ is not a neighbor of $v$, it is not identified with any vertex of $Z_{i}^{\prime}-v^{\prime}$, so $Z_{i}^{\prime \prime}$ is a cycle, and of the same length as $Z_{i}^{\prime}$. Thus, $\Phi^{\prime \prime}$ is closed-2-cell.

Since all faces of type (1) are $C_{4}$-faces, mapping each non- $C_{4}$-face $Z_{i}^{\prime}$ to $Z_{i}^{\prime \prime}$ and each non- $C_{4}$-face of type (3) to itself gives the required degree-preserving bijection for non- $C_{4}$-faces.

Let $m_{s t}$ be the maximum number of edges shared by a face of type $(s)$ and a distinct face of type $(t)$. Clearly $m_{13}=0$; since $\Phi$ is face-simple, $m_{11}, m_{12} \leq 1$; and by the hypothesis on $\Phi^{\prime}$, $m_{32}, m_{33} \leq 1$. Consider two arbitrary distinct faces $Z_{i}^{\prime \prime}, Z_{j}^{\prime \prime}$ of type (2). Suppose $Z_{i}-v$ and $Z_{j}-v$ share an edge $a b$. Since $\Phi$ is simple and $\delta(\Phi) \geq 3$, we cannot have $Z_{i}=(v a b c)$ and $Z_{j}=(v a b d)$, so we may assume that we have $Z_{i}=(v a b c)$ and $Z_{j}=(v b a d)$. But then $a$ and $b$ are adjacent neighbors of $v$, a contradiction. Therefore, $Z_{i}^{\prime \prime}$ and $Z_{j}^{\prime \prime}$ share no edges of $G-v$, and by the hypothesis on $\Phi^{\prime}$ they share at most one edge of $G^{\prime}-v^{\prime}$, so $m_{22} \leq 1$, and $\Phi^{\prime \prime}$ is face-simple.

\subsection{Graphical surfaces}

White [33] showed that any composition $G\left[\overline{K_{2}}\right]$, where $G$ is a simple graph without isolated vertices, has an orientable quadrangular embedding. Craft $[5,7]$ developed graphical surfaces, which yield a simple proof of this result. We outline his proof, since we need his construction in Section 6 .

For a graph $G$, the graphical surface $S(G)$ derived from $G$ is a surface obtained from an embedding of $G$ in $\mathbb{R}^{3}$ by blowing up every vertex $u$ into a sphere $\Sigma_{u}$ and replacing every edge $u v$ by a tube $T_{u v}$ joining the spheres $\Sigma_{u}$ and $\Sigma_{v}$. Since we work in $\mathbb{R}^{3}$, the resulting surface $S(G)$ is orientable.

Lemma 3.6 (Craft $[5,7]$ ). Let $G$ be a connected simple graph. Then $G\left[\overline{K_{2}}\right]$ has a quadrangular embedding in the graphical surface $S(G)$.

Outline of proof. We embed $G\left[\overline{K_{2}}\right]$ in $S(G)$ as follows. For any vertex $u \in V(G)$, let $u_{\mathrm{N}}$ (north pole) and $u_{\mathrm{S}}$ (south pole) be two points in the sphere $\Sigma_{u}$; they represent the two vertices of $G\left[\overline{K_{2}}\right]$ corresponding to $u$. We may assume that all tubes joined to $\Sigma_{u}$ are joined in some cyclic order around the equator of $\Sigma_{u}$. There are four edges of $G\left[\overline{K_{2}}\right]$ corresponding to each $u v \in E(G)$, which are $u_{\mathrm{N}} v_{\mathrm{N}}, u_{\mathrm{N}} v_{\mathrm{S}}, u_{\mathrm{S}} v_{\mathrm{S}}$ and $u_{\mathrm{S}} v_{\mathrm{N}}$. These can all be embedded along $T_{u v}$ (there are two different ways to do this, but for our purposes it will not matter which is used). In the resulting embedding, every edge is contained in two quadrilaterals. For example, $u_{\mathrm{S}} v_{\mathrm{N}}$ is contained in quadrilaterals $Q_{u}=\left(t_{X} u_{\mathrm{N}} v_{\mathrm{N}} u_{\mathrm{S}}\right)$ and $Q_{v}=\left(u_{\mathrm{S}} v_{\mathrm{N}} w_{Y} v_{\mathrm{S}}\right)$ where $t u, v w \in E(G)$ and $X, Y \in\{\mathrm{N}, \mathrm{S}\}$. Note that if $u$ has degree 1 , then $t_{X}=v_{\mathrm{S}}$.

White and Craft dealt only with orientable embeddings. However, we can also produce nonorientable embeddings. Given a graphical surface, we can replace a tube $T_{u v}$ by a twisted tube $\widetilde{T}_{u v}$ by 
taking a simple closed curve $\gamma$ around $T_{u v}$ with a specified positive direction, cutting along it to produce two boundary curves $\gamma_{1}$ and $\gamma_{2}$, then re-identifying $\gamma_{1}$ with $\gamma_{2}$ so that the positive direction along $\gamma_{1}$ corresponds to the negative direction along $\gamma_{2}$ (this cannot be done in $\mathbb{R}^{3}$ ). We can still embed the four edges between $\left\{u_{\mathrm{N}}, u_{\mathrm{S}}\right\}$ and $\left\{v_{\mathrm{N}}, v_{\mathrm{S}}\right\}$ along $\widetilde{T}_{u v}$; they become orientation-reversing edges relative to the original orientation at each vertex. Depending on which tubes we replace, the resulting embedding may be nonorientable.

Lemma 3.7. Let $G$ be a connected simple graph with at least one cycle. Then $G\left[\overline{K_{2}}\right]$ has a quadrangular embedding in a nonorientable modified graphical surface $\widetilde{S}(G)$.

Proof. Choose one edge $u v$ belonging to a cycle and replace the tube $T_{u v}$ by a twisted tube $\widetilde{T}_{u v}$ in the construction of Lemma 3.6. The resulting embedding is nonorientable because the cycle (uvw ...z) in $G$ gives an orientation-reversing cycle $\left(u_{\mathrm{N}} v_{\mathrm{N}} w_{\mathrm{N}} \ldots z_{\mathrm{N}}\right)$ in the embedding of $G\left[\overline{K_{2}}\right]$ in the new surface $\widetilde{S}(G)$.

\subsection{Voltage graphs}

We assume the reader is familiar with voltage graph constructions for embeddings. We summarize the main features; see [11] for more details.

Given a graph $G$, assign an arbitrary plus direction to each edge. A function $\alpha$ from the plusdirected edges of $G$ to a group $\Gamma$ is an ordinary voltage assignment on $G$. The pair $\langle G, \alpha\rangle$ is called an ordinary voltage graph. The derived graph $G^{\alpha}$ has vertex set $V(G) \times \Gamma$ and an edge from $u_{a}=(u, a)$ to $v_{b}=(v, b)$ whenever $u v$ is a plus-directed edge in $G$ and $b=a \cdot \alpha(u v)$.

If $G$ has an embedding $\Phi$, represented by a rotation of edges at each vertex and edge signatures, then $G^{\alpha}$ has a derived embedding $\Phi^{\alpha}$ : for each $u_{a} \in V\left(G^{\alpha}\right)$ use the natural bijection between edges incident with $u$ in $G$ and edges incident with $u_{a}$ in $G^{\alpha}$ to define the rotation at $u_{a}$ from the rotation at $u$, and give each edge $u_{a} v_{b}$ of $G^{\alpha}$ the signature of the corresponding edge $u v$ in $G$.

For each walk $W=v_{0} e_{1} v_{1} e_{2} v_{2} \ldots e_{k} v_{k}$ in $G$ define its total voltage to be $\alpha\left(e_{1}\right)^{\epsilon_{1}} \alpha\left(e_{2}\right)^{\epsilon_{2}} \ldots \alpha\left(e_{k}\right)^{\epsilon_{k}}$ where $\epsilon_{i}$ is +1 if $W$ uses $e_{i}$ in the plus direction and -1 otherwise. The faces of $\Phi^{\alpha}$ come from the faces of $\Phi$ : each face in $\Phi$ with degree $k$ whose boundary walk has total voltage of order $r$ in $\Gamma$ yields $|\Gamma| / r$ faces of degree $k r$ in $\Phi^{\alpha}$. Also, $\Phi^{\alpha}$ is nonorientable if and only if $\Phi$ is nonorientable and has an orientation-reversing closed walk whose total voltage is the identity of $\Gamma$.

\section{Embeddings from diamond sums}

In this section we prove Theorems 1.2 and 1.3 by constructing embeddings of minimum genus with all face degrees at least 4 , and with all face degrees even, for each complete graph $K_{n}, n \geq 4$. Our constructions are inductive. The base cases are provided in Appendix A.

The induction steps use quadrangular embeddings of complete bipartite graphs and of $K_{7}^{+}$and $K_{11}^{+}$, where $K_{n}^{+}$denotes the graph obtained from $K_{n}$ by subdividing an edge. In Figure 1 we provide embeddings $\widetilde{\Psi}_{7}$ of $K_{7}$ in $N_{5}$ (as a polygon with labeled vertices, indicating how edges are to be identified around the boundary) and $\Psi_{11}$ of $K_{11}$ in $S_{9}$ (as a rotation system; see [11, Section 
3.2]). Each embedding is face-simple and all faces are $C_{4}$-faces apart from two $C_{3}$-faces that share an edge $x y\left(x y=01\right.$ for $\widetilde{\Psi}_{7}$ and $x y=56$ for $\left.\Psi_{11}\right)$. Nonorientability of $\widetilde{\Psi}_{7}$ follows from the fact that there are edges, such as 05 , used twice in the same direction around the outer boundary of the polygon. By subdividing $x y$ with a vertex $z$ in each case, we obtain embeddings $\widetilde{\Psi}_{7}^{+}$and $\Psi_{11}^{+}$of graphs $K_{7}^{+}$and $K_{11}^{+}$. These embeddings are not face-simple, but with the choice $v^{\prime}=x$ they satisfy the hypotheses for $\Phi^{\prime}$ in Lemma 3.5.
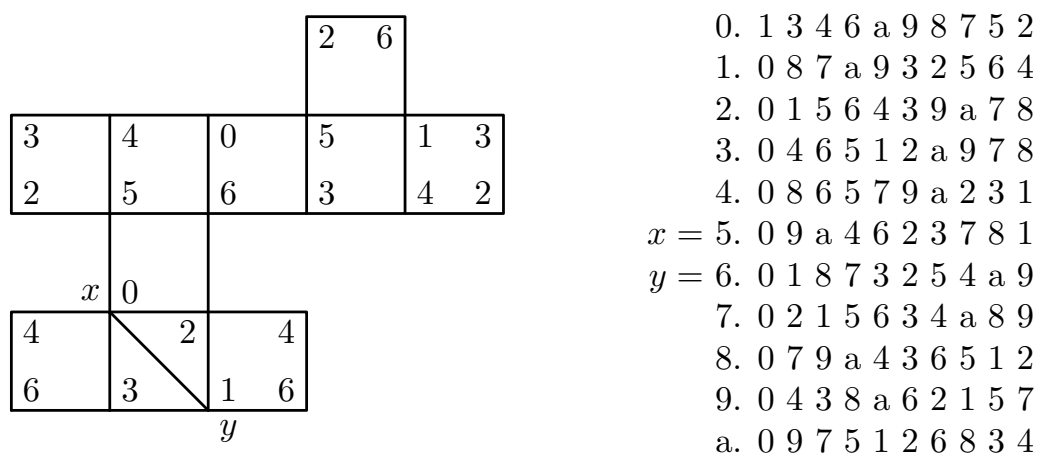

Figure 1: Embeddings $\widetilde{\Psi}_{7}$ of $K_{7}$ in $N_{5}$ (left) and $\Psi_{11}$ of $K_{11}$ in $S_{9}$ (right).

As part of determining the orientable and nonorientable genera of $K_{m, n}$, Ringel showed that $K_{m, n}$ has quadrangular embeddings in certain cases. Bouchet [3] later provided a simpler proof.

Lemma 4.1 (Ringel $[29,30])$. The complete bipartite graph $K_{m, n}$ has an orientable quadrangular embedding whenever $(m-2)(n-2) \equiv 0(\bmod 4)$ and $\min \{m, n\} \geq 2$, and a nonorientable quadrangular embedding whenever $m n \equiv 0(\bmod 2)$ and $\min \{m, n\} \geq 3$.

First we consider nonorientable embeddings. The following two lemmas provide the induction steps in our proof.

Lemma 4.2. If a complete graph $K_{n}, n \geq 4$, admits a face-simple orientable or nonorientable quadrangular embedding, then $K_{n+4}$ admits a face-simple nonorientable quadrangular embedding.

Proof. Take $K_{7}^{+}$as described above, with special vertices $x, y, z$. We may interpret $K_{7}^{+}$as a join $\left(K_{1} \cup K_{5}\right)+\overline{K_{2}}$ where $z$ is the vertex of the $K_{1}$, and $x$ and $y$ are the vertices of the $\overline{K_{2}}$. We build the embedding of $K_{n+4}$ in two steps from the embedding $\Phi_{1}=\widetilde{\Psi}_{7}^{+}$of $K_{7}^{+}$as described above, an orientable or nonorientable quadrangular embedding $\Phi_{2}$ of $K_{6, n-1}$ from Lemma 4.1, and the assumed quadrangular embedding $\Phi_{3}$ of $K_{n}$.

Let $x^{\prime}$ be a vertex of $K_{6, n-1}$ of degree 6 . Since $\delta\left(\Phi_{2}\right)=\min (6, n-1) \geq 3, K_{6, n-1}$ is bipartite, and using Observation 3.4, we satisfy the hypotheses for $\Phi$ in Lemma 3.5 by taking $\Phi=\Phi_{2}$ and $v=x^{\prime}$. From above we also satisfy the hypotheses for $\Phi^{\prime}$ in Lemma 3.5 by taking $\Phi^{\prime}=\Phi_{1}=\widetilde{\Psi}_{7}^{+}$ and $v^{\prime}=x$. Therefore, by Lemma 3.5, applying the diamond sum to $\Phi_{1}$ and $\Phi_{2}$ at $x$ and $x^{\prime}$ yields a face-simple quadrangulation $\Phi_{12}$, which is nonorientable since $\Phi_{2}$ is nonorientable. The underlying graph $G_{12}=K_{7}^{+} \diamond K_{6, n-1}$ is $\left(K_{1} \cup K_{5}\right)+\overline{K_{n-1}}$, where $z$ is the vertex of the $K_{1}$, with degree $n-1$, and $y$ is now a vertex of the $\overline{K_{n-1}}$. 
Since $\delta\left(\Phi_{12}\right)=\min (6, n-1) \geq 3$ and the neighbors of $z$ in $G_{12}$ are independent, we satisfy the hypotheses for $\Phi$ in Lemma 3.5 by taking $\Phi=\Phi_{12}$ and $v=z$. Let $z^{\prime}$ be a vertex of $K_{n}$. We also satisfy the hypotheses for $\Phi^{\prime}$ in Lemma 3.5 by taking $\Phi^{\prime}=\Phi_{3}$ and $v^{\prime}=z^{\prime}$. Therefore, by Lemma 3.5, applying the diamond sum to $\Phi_{12}$ and $\Phi_{3}$ at $z$ and $z^{\prime}$ yields a face-simple quadrangulation $\Phi_{123}$, which is nonorientable since $\Phi_{12}$ is nonorientable. The underlying graph $\left(\left(K_{1} \cup K_{5}\right)+\overline{K_{n-1}}\right) \diamond K_{n}$ is $K_{5}+K_{n-1}=K_{n+4}$.

The same proof also shows the following. The $C_{p}$-face in the embedding $\Phi_{3}$ of $K_{n}$ corresponds to a $C_{p}$-face in the embedding $\Phi_{123}=\Phi_{12} \diamond \Phi_{3}$ of $K_{n+4}$ by the degree-preserving bijection of Lemma 3.5 .

Lemma 4.3. Suppose that $n \geq p \geq 5$. If a complete graph $K_{n}$ admits a face-simple orientable or nonorientable embedding in which all faces are $C_{4}$-faces except for one $C_{p}$-face, then $K_{n+4}$ has a face-simple nonorientable embedding in which all faces are $C_{4}$-faces except for one $C_{p}$-face.

Theorem 4.4. Given an integer $n$, let $k=2+\lceil n(n-5) / 4\rceil$.

Suppose that $n \geq 6$. If $n \equiv 0$ or $1(\bmod 4)$ then $K_{n}$ has a face-simple quadrangular embedding in $N_{k}$. If $n \equiv 2$ or $3(\bmod 4)$ then $K_{n}$ has a face-simple embedding in $N_{k}$ in which every face is a $\mathrm{C}_{4}$-face except for one $C_{6}$-face.

For $n=4, K_{4}$ has a quadrangular embedding in $N_{k}=N_{1}$ that is closed-2-cell but not face-simple. For $n=5, K_{5}$ has no quadrangular embedding in $N_{k}=N_{2}$, but has an embedding in $N_{3}$ with three $C_{4}$-faces and one 8-face.

Proof. In each case the genus will follow by simple face/edge counting and Euler's formula, so we focus on the other properties. For $n \not \equiv 1(\bmod 4)$, Appendix A gives the required embeddings $\widetilde{\Theta}_{n}$ for $n \in\{4,6,7,8\}$, and we then repeatedly apply Lemma 4.2 or 4.3 . (We need $n=8$ because the embedding for $n=4$ is not face-simple.)

Suppose that $n \equiv 1(\bmod 4)$. For $n=5$, Hutchinson [16, proof of Theorem 2] and Hartsfield and Ringel [15, Theorem 2] showed that there is no quadrangular embedding of $K_{5}$ in the Klein bottle $N_{2}$. However, there is a face-simple quadrangular embedding $\widetilde{\Psi}_{6}^{-}$of $K_{6}-e$ in $N_{3}$ given in Appendix $\mathrm{A}$, and deleting vertex 0 gives the required embedding of $K_{5}$ in $N_{3}$. For $n \geq 9$, applying Lemma 4.2 to the orientable quadrangular embedding $\Theta_{5}$ of $K_{5}$ from Appendix A gives a nonorientable quadrangular embedding of $K_{9}$, and we then repeatedly apply Lemma 4.2.

Theorem 1.3 follows because every embedding given in Theorem 4.4 is even-faced.

By adding chords (carefully, for $K_{5}$ ) or a single vertex inside the face of degree greater than 4 we also obtain the following.

Corollary 4.5. Suppose that $n \geq 4$ and $k=\lceil n(n-5) / 4\rceil+2$. If $n \equiv 0$ or $1(\bmod 4)$ and $n \neq 5$, then $K_{n}$ has a quadrangular embedding in $N_{k}$, which is face-simple if $n \geq 8$. For $n=5, K_{5}$ is a subgraph of a quadrangular 5-vertex embedding with multiple edges in $N_{3}$, and of a face-simple simple 6-vertex quadrangular embedding in $N_{3}$. If $n \equiv 2$ or $3(\bmod 4), K_{n}$ is a subgraph of a quadrangular $n$-vertex embedding with multiple edges in $N_{k}$, and of a face-simple simple $(n+1)$-vertex quadrangular embedding in $N_{k}$. 
Proof. For $n=5$ we take $\widetilde{\Psi}_{6}^{-}$from Appendix A as the simple 6-vertex embedding. Deleting vertex 0 from $\widetilde{\Psi}_{6}^{-}$leaves an 8 -face $\left(1_{1} 4_{1} 5_{1} 21_{2} 34_{2} 5_{2}\right)$ (subscripting occurrences of the same vertex to distinguish them). We can add multiple edges $1_{1} 3$ and $5_{1} 3$.

Now we turn to orientable embeddings. The following two lemmas provide the induction steps in our proof. They are proved in exactly the same way as Lemmas 4.2 and 4.3 , except that we take $\Phi_{1}$ to be the orientable quadrangular embedding $\Psi_{11}^{+}$of $K_{11}^{+}$instead of the nonorientable embedding $\widetilde{\Psi}_{7}^{+}$ of $K_{7}^{+}$, and $\Phi_{2}$ to be an orientable quadrangular embedding of $K_{10, n-1}$, instead of a nonorientable embedding of $K_{6, n-1}$.

Lemma 4.6. If a complete graph $K_{n}, n \geq 4$, admits a face-simple orientable quadrangular embedding, then $K_{n+8}$ admits a face-simple orientable quadrangular embedding.

Lemma 4.7. Suppose that $5 \leq p \leq n$. If a complete graph $K_{n}$ admits a face-simple orientable embedding in which all faces are $C_{4}$-faces except for one $C_{p}$-face, then $K_{n+8}$ has a face-simple orientable embedding in which all faces are $C_{4}$-faces except for one $C_{p}$-face.

There is one case where we cannot find an embedding with all of the properties we would like.

Proposition 4.8. Every general embedding of $K_{6}$ in $S_{2}$ is cellular with five $C_{4}$-faces and two $C_{5}$ faces, and such an embedding exists. Thus, $K_{6}$ has no general even-faced embedding in $S_{2}$.

Outline of proof. Let $\Phi$ be a general embedding of $K_{6}$ in $S_{2}$, with $n=6$ vertices, $m=15$ edges, $r$ faces, and $r_{i}$ faces of degree $i$. By Euler's inequality, $r \geq \varepsilon-n+m=-2-6+15=7$. By Observation 3.3, $\delta^{*}(\Phi) \geq 4$, and so $30=2 m=4 r_{4}+5 r_{5}+6 r_{6}+\ldots \geq 4 r$. Hence $r=7$, with either $r_{4}=6$ and $r_{6}=1$, or $r_{4}=5$ and $r_{5}=2$. Since $r=7, \Phi$ is cellular. By Observation 3.2 the 4 -faces and any 5 -faces are bounded by cycles; any 6 -face is bounded by a cycle or a 'bowtie' walk (abcade).

Now that we have restricted the structure of $\Phi$, we can perform a case analysis to show that the embedding is as described. Details may be found in Appendix B. It is also easy to generate and check all rotation systems for $K_{6}$ (up to isomorphism) by computer. We did this; it ran in less than a minute. The embedding $\Theta_{6}$ from Appendix A demonstrates existence.

Theorem 4.9. Given an integer $n$, let $h=1+\lceil n(n-5) / 8\rceil$.

Suppose that $n=5$ or $n \geq 7$. If $n \equiv 0$ or $5(\bmod 8)$, then $K_{n}$ has a face-simple quadrangular embedding in $S_{h}$. If $n \neq \equiv 0$ and $5(\bmod 8)$ then $K_{n}$ has a face-simple embedding in which every face is a $C_{4}$-face except for one $C_{p}$-face, where $p \in\{6,8,10\}$ (specifically, $p=12-(n(n-5) \bmod 8)$ ).

For $n=4, K_{4}$ has an embedding in $S_{h}=S_{1}$ with one $C_{4}$-face and one 8-face. For $n=6, K_{6}$ has no even-faced embedding in $S_{h}=S_{2}$, but has an embedding in $S_{2}$ with five $C_{4}$-faces and two $C_{5}$-faces, and an embedding in $S_{3}$ with four $C_{4}$-faces and one 14-face.

Proof. In each case the genus will follow by simple face/edge counting and Euler's formula, so we focus on the other properties. For $n \neq 4$ and 6 , Appendix A gives the required embeddings $\Theta_{n}$ of $K_{n}$ for $n \in\{5,7,8,9,10,11,12,14\}$, covering all classes modulo 8 , and we then repeatedly apply Lemma 4.6 or 4.7. For $n=4$, there cannot be an embedding with a $C_{8}$-face, but an embedding $\Theta_{4}$ with an 8-face is given in Appendix A. 
For $n=6$, see Proposition 4.8. To obtain the embedding of $K_{6}$ in $S_{3}$ with a 14 -face, take $\Theta_{6}$ from Appendix A and swap the positions of 1 and 2 in the rotation of vertex 0 . This replaces face boundaries (01234), (03142) and (0251) by a single closed walk (01234025103142) of length 14.

Theorem 1.2 follows because every embedding given in Theorem 4.9, except for the embedding of $K_{6}$ in $S_{2}$, is even-faced.

By adding chords (carefully, for $K_{4}$ and $K_{6}$ ) or a single vertex (or two vertices, for $K_{6}$ ) inside the face of degree greater than 4 we also obtain the following.

Corollary 4.10. Suppose that $n \geq 4$ and $h=\lceil n(n-5) / 8\rceil+1$. If $n \equiv 0$ or $5(\bmod 8)$, then $K_{n}$ has a quadrangular embedding in $S_{h}$. If $n \neq \equiv 0$ and $5(\bmod 8)$ and $n \neq 6, K_{n}$ is a subgraph of a quadrangular n-vertex embedding with multiple edges in $S_{h}$, and of a simple $(n+1)$-vertex quadrangular embedding in $S_{h}$. For $n=6, K_{6}$ is a subgraph of a quadrangular 6-vertex embedding with multiple edges in $S_{3}$ and a simple 8-vertex quadrangular embedding in $S_{3}$.

Proof. For $K_{4}$, the 8 -face in $\Theta_{4}$ is $\left(0_{1} 1_{1} 2_{1} 3_{1} 1_{2} 0_{2} 3_{2} 2_{2}\right)$ (subscripting occurrences of the same vertex to distinguish them) and we can add multiple edges $0_{1} 3_{1}, 1_{2} 2_{2}$. Adding a new vertex 4 adjacent to $0_{1}, 2_{1}, 1_{2}, 3_{2}$ gives an embedding isomorphic to the the quadrangular embedding $\Theta_{5}$ of $K_{5}$ in $S_{1}$.

For $K_{6}$, the 14 -face from the proof of Theorem 4.9 is $\left(0_{1} 1_{1} 2_{1} 3_{1} 4_{1} 0_{2} 2_{2} 51_{2} 0_{3} 3_{2} 1_{3} 4_{2} 2_{3}\right)$ and we can add multiple edges $0_{1} 3_{1}, 3_{1} 2_{2}, 3_{1} 4_{2}, 2_{2} 0_{3}, 0_{3} 4_{2}$ or new vertices 6 adjacent to $2_{3}, 1_{1}, 3_{1}, 0_{2}, 5$ and then 7 adjacent to $1_{2}, 3_{2}, 4_{2}, 6$. (Or the 8 -vertex simple quadrangulation of $S_{3}$ containing $K_{7}$ also contains $\left.K_{6} \cdot\right)$

\section{Proof of the Even Map Color Theorem}

The Map Color Theorem says that for a graph embedding $\Phi$ in a surface $\Sigma \neq S_{0}, \chi(\Phi) \leq H(\Sigma)=$ $\lfloor(7+\sqrt{49-24 \varepsilon(\Sigma)}) / 2\rfloor$ (the Heawood number of $\Sigma)$, which can be improved to $\chi(\Phi) \leq H(\Sigma)-1$ if $\Sigma=N_{2}$, and these bounds are sharp. Hutchinson showed that this can be significantly improved if the embedding is even-faced. In this section we strengthen her bound in one case, and use the embeddings constructed in Section 4 to show that the bounds are sharp.

Theorem 5.1 (Hutchinson [16, Theorems 1 and 2 and Corollary 2]). For a surface $\Sigma$ define $H_{\text {even }}(\Sigma)=\lfloor(5+\sqrt{25-16 \varepsilon(\Sigma)}) / 2\rfloor$. If $\Phi$ is an even-faced graph embedding in a surface $\Sigma \neq S_{0}$ then $\chi(\Phi) \leq H_{\text {even }}(\Sigma)$. If $\Sigma=N_{2}$ this can be improved to $\chi(\Phi) \leq H_{\text {even }}\left(N_{2}\right)-1=4$. These results are sharp when $\Sigma=N_{1}, N_{2}$ or $S_{1}$.

Hutchinson's proof requires $\Phi$ to be cellular, because she first proves a face-coloring result and applies that to the dual $\Phi^{*}$; each vertex of $\Phi$ needs to correspond to a distinct face of $\Phi^{*}$. One way to extend the result to general embeddings is by first applying the following lemma.

Lemma 5.2. Suppose $\Phi$ is a general embedding. Then we can construct a new embedding $\Phi^{\prime}$ in the same surface by adding edges, such that (a) $\Phi^{\prime}$ is cellular, (b) each face of $\Phi$ corresponds to a distinct face of $\Phi^{\prime},(c)$ if two faces are adjacent in $\Phi$ then the corresponding faces are adjacent in $\Phi^{\prime}$, (d) if $\Phi$ is even-faced then so is $\Phi^{\prime}$, and (e) if $\Phi$ is even-vertexed then so is $\Phi^{\prime}$. 
Proof. We can add edges inside faces to destroy each internal handle or crosscap (run an edge along the handle or across the crosscap) and connect different boundary components without creating any new faces, satisfying (a)-(d). If we replace each new edge by two parallel edges bounding a 2-face we still satisfy (a)-(d) and also satisfy (e). In particular, since new edges have the same face on both sides, this does not violate (c).

We can also prove Theorem 5.1 directly for general embeddings. We outline a proof of the general inequality in Theorem 5.1, based on translating and simplifying the proof of [16, Theorem $1]$, as we need some details later. We use the following preliminary results, which are implicit in the arguments of [16].

Observation 5.3. If we remove edges or vertices from an even-faced embedding it remains evenfaced.

Lemma 5.4. If $\Psi$ is a general $n$-vertex embedding in a surface $\Sigma$ with average (or minimum) vertex degree at least $d$ and average (or minimum) face degree at least 4 , then $n(d-4) \leq-4 \varepsilon(\Sigma)$.

Proof. Suppose $\Psi$ has $m$ edges and $r$ faces, and let $\varepsilon=\varepsilon(\Sigma)$. By Euler's inequality $n-m+r \geq \varepsilon$, and the degree conditions mean that $2 m \geq n d$ and $2 m \geq 4 r$. Thus,

$$
4 \varepsilon \leq 4 n-4 m+4 r=4 n-2 m+(4 r-2 m) \leq 4 n-n d+0=n(4-d)
$$

and the result follows.

Lemma 5.5. Let $\Sigma$ be a surface with $\Sigma \neq S_{0}$, and let $d=H_{\text {even }}(\Sigma)$. Then $d \geq 4$ and $d$ is the smallest positive integer such that $(d+1)(d-4)>-4 \varepsilon(\Sigma)$.

Proof. Consider $p(x)=(x+1)(x-4)+4 \varepsilon(\Sigma)=x^{2}-3 x-4+4 \varepsilon(\Sigma)$. Since $\varepsilon(\Sigma) \leq 1, p(1)=$ $p(2)<0$, and so $p(x)$ has two real roots $\alpha_{1}<1$ and $\alpha_{2}>2$. By the quadratic formula, $\alpha_{2}=$ $(3+\sqrt{25-16 \varepsilon(\Sigma)}) / 2$. A positive integer $d$ has $p(d)>0$ if and only if $d>\alpha_{2}$. But since $d$ is an integer, $d>\alpha_{2}$ is equivalent to $d \geq\left\lfloor\alpha_{2}+1\right\rfloor=H_{\text {even }}(\Sigma)$. So the smallest positive integer $d$ with $(d+1)(d-4)>-4 \varepsilon(\Sigma)$, or $p(d)>0$, or $d>\alpha_{2}$, is exactly $H_{\text {even }}(\Sigma)$.

Since $\varepsilon(\Sigma) \leq 1$, if $d=H_{\text {even }}(\Sigma)$ then the formula for $H_{\text {even }}(\Sigma)$ yields $d \geq 4$.

Outline of proof that $\chi(\Phi) \leq H_{\text {even }}(\Sigma)$ for general embeddings. We label the steps here for reference. Let $\varepsilon=\varepsilon(\Sigma) \leq 1$. (A) Let $d=H_{\text {even }}(\Sigma) \geq 4$. (B) Let $\Phi$ be an even-faced embedding of minimum order $n$ in $\Sigma$ whose graph $G$ is not $d$-colorable. (C) If $n \leq d$, then $G$ is $d$-colorable, so $n \geq d+1$. (D) Remove all loops from $\Phi$ to obtain an even-faced embedding $\Phi_{1}$ of $G_{1}$ with $\chi\left(G_{1}\right)=\chi(G)$. (E) Starting with $\Phi_{1}$ repeatedly remove one edge from each 2-face until no 2-faces remain, giving an even-faced embedding $\Phi_{2}$ of a graph $G_{2}$ with $\chi\left(G_{2}\right)=\chi(G)$ and $\delta^{*}\left(\Phi_{2}\right) \geq 4$. (F) If $\delta\left(\Phi_{2}\right) \leq d-1$, then we can remove a vertex $x$ of degree at most $d-1, d$-color $G_{2}-x$ by minimality since $\Phi_{2}-x$ remains even-faced, then color $x$, so $G_{2}$, and hence $G$, is $d$-colorable. Thus, $\delta\left(\Phi_{2}\right) \geq d$. (G) By (E), $(\mathrm{F})$, Lemma 5.4 and $(\mathrm{C}),(d+1)(d-4) \leq n(d-4) \leq-4 \varepsilon$, contradicting Lemma 5.5.

Working with general embeddings, rather than cellular embeddings, simplifies the above proof in step $(\mathrm{F})$ : we do not have to worry about losing cellularity when we delete a vertex. 

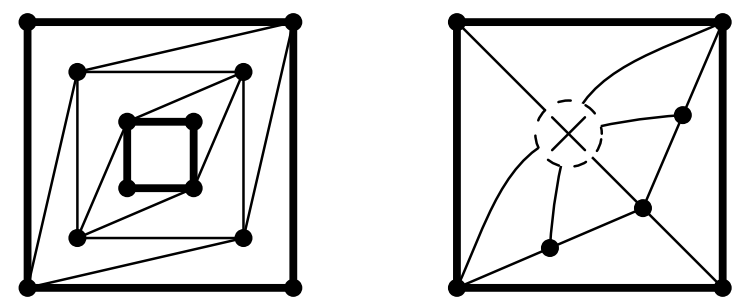

Figure 2: Adding a quadrangular handle (left) or crosscap (right).

We now show that the bound of Theorem 5.1 can be improved by 1 when the surface is $S_{2}$, and then show that with this improvement the result is sharp.

Proposition 5.6. Suppose $G$ is a graph (multiple edges and loops allowed) with a general even-faced embedding $\Phi$ in $S_{2}$. Then (ignoring loops when coloring) $\chi(G) \leq H_{\text {even }}\left(S_{2}\right)-1=5$.

Proof. We modify the above proof that $\chi(\Phi) \leq H_{\text {even }}(\Sigma)$. In (A) take $d=5$ instead of $d=$ $H_{\text {even }}\left(S_{2}\right)=6$. As in (B), let $\Phi$ be an even-faced embedding of minimum order $n$ in $\Sigma=S_{2}$ $(\varepsilon=-2)$ of a graph $G$ with $\chi(G)>d=5$. Apply Lemma 5.2 to convert $\Phi$ into an even-faced cellular embedding $\Phi_{0}$ of a necessarily connected graph $G_{0}$. Apply (D) and (E) to $\Phi_{0}$ to construct $\Phi_{1}$ and $\Phi_{2}$ as above. Then $G_{2}$ is loopless and connected, $\chi\left(G_{2}\right)=\chi\left(G_{1}\right)=\chi\left(G_{0}\right) \geq \chi(G)$, and $\delta^{*}\left(\Phi_{2}\right) \geq 4$. From (C) and (F), $n \geq d+1=6$ and $\delta\left(\Phi_{2}\right) \geq d=5$.

Delete edges from $\Phi_{2}$ to obtain an even-faced embedding $\Phi_{3}$ in $S_{2}$ of an underlying connected simple graph $G_{3}$ of $G_{2}$. We have $\chi\left(G_{3}\right)=\chi\left(G_{2}\right) \geq \chi(G)>5$. By the argument of $(\mathrm{F})$, we still have $\delta\left(\Phi_{3}\right) \geq 5$. Let $m$ and $r$ be the number of edges and faces of $\Phi_{3}$, respectively.

Suppose that $n=6$. If $G_{3} \neq K_{6}$, then $G_{3}$, and hence $G$, is 5-colorable, so $G_{3}=K_{6}$. But then, by Proposition $4.8, \Phi_{3}$ cannot exist, a contradiction.

So $n \geq 7$. If $\Delta\left(G_{3}\right)=5$, then since $G_{3}$ is simple, connected and not equal to $K_{6}$, by Brooks' Theorem, $G_{3}$, and hence $G$, is 5 -colorable. Thus, $G_{3}$ has a vertex of degree 6 or more, from which $2 m \geq 5(n-1)+6=5 n+1$, so $m \geq\lceil(5 n+1) / 2\rceil$. By Observation $3.3, \delta^{*}\left(\Phi_{3}\right) \geq 4$, so $2 m \geq 4 r$ and $r \leq m / 2$. Therefore, $-2=\varepsilon \leq n-m+r \leq n-m+m / 2=n-m / 2 \leq n-\lceil(5 n+1) / 2\rceil / 2$. This fails if $n \geq 8$, so $n=7$. Moreover, when $n=7$ this is tight, so all steps in our reasoning are tight. In particular, $G_{3}$ has one vertex of degree 6 and $n-1=6$ vertices of degree 5 . But then $G_{3}=K_{7}-3 K_{2}$ (delete three independent edges from $K_{7}$ ), which is 4-colorable, a contradiction.

So no such $\Phi$ exists, and $\chi(G) \leq 5$ for all $G$ with an even-faced embedding in $S_{2}$.

Before proving the main result of this section, we introduce two operations. By adding a quadrangular handle to an orientable quadrangulation $\Phi$, we mean deleting two distinct faces, and inserting a handle (cylinder or annulus) with four new vertices as shown at left in Figure 2, identifying the inner and outer 4-cycles with the boundaries of the deleted faces, so that the resulting quadrangular embedding $\Phi^{\prime}$ is still orientable. Note that if $\Phi$ is simple, so is $\Phi^{\prime}$, and if $\Phi$ is face-simple, so is $\Phi^{\prime}$.

Also, by adding a quadrangular crosscap to a quadrangular embedding $\Phi$ we mean inserting a crosscap and three new vertices in a face, as shown at right in Figure 2. The resulting quadrangular embedding $\Phi^{\prime}$ is nonorientable. If $\Phi$ is simple, so is $\Phi^{\prime}$, and if $\Phi$ is face-simple, so is $\Phi^{\prime}$. 

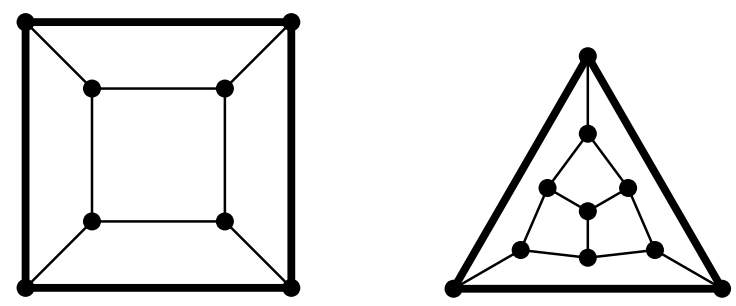

Figure 3: Adding new faces in the same surface.

In both cases, $\chi\left(\Phi^{\prime}\right) \geq \chi(\Phi)$.

Proof of Theorem 1.4, the Even Map Color Theorem. Consider first the vertex-coloring result (a). The upper bound on $\chi(\Phi)$ follows from Hutchinson's Theorem 5.1, our Proposition 5.6, and the wellknown fact that graphs with even-faced embeddings in the plane are bipartite. Hutchinson provided sharpness examples for $\Sigma=N_{1}, N_{2}$ and $S_{1}$, but we now provide sharp quadrangular examples for all surfaces.

First we apply the results of Section 4. For $n \geq 7$, it follows from Corollaries 4.5 and 4.10 and addition of quadrangular handles or quadrangular crosscaps that there is a face-simple quadrangular embedding $\Omega_{n, \Sigma}$ in $\Sigma$ of a simple graph with $K_{n}$ as a subgraph, and hence with $\chi\left(\Omega_{n, \Sigma}\right) \geq n$, provided $\varepsilon(\Sigma) \leq n(5-n) / 4$, i.e., $n(n-5) \leq-4 \varepsilon(\Sigma)$. This also works for $n=5$ if $\Sigma$ is orientable and for $n=6$ if $\Sigma$ is nonorientable.

Now suppose that $\varepsilon(\Sigma) \leq-4$, and let $d=c(\Sigma)=H_{\text {even }}(\Sigma) \geq 7$. By Lemma 5.5, $d$ is the smallest positive integer with $(d+1)(d-4)>-4 \varepsilon(\Sigma)$, so this inequality fails with $d$ replaced by $d-1$, giving $d(d-5) \leq-4 \varepsilon(\Sigma)$. Hence, by the previous paragraph, there is an embedding $\Omega_{d, \Sigma}$ with $d \leq \chi\left(\Omega_{d, \Sigma}\right) \leq H_{\text {even }}(\Sigma)=d$, which provides the required sharpness example.

The remaining surfaces are $S_{h}$ for $0 \leq h \leq 2$ and $N_{k}$ for $1 \leq k \leq 5$. For $S_{0}$ take the standard planar (spherical) embedding of the cube. If $\Sigma=S_{1}$ or $S_{2}$ take $\Omega_{5, \Sigma}$, which has $5 \leq \chi\left(\Omega_{5, \Sigma}\right) \leq$ $c(\Sigma)=5$. For $N_{1}$ and $N_{2}$, take $\widetilde{\Theta}_{4}$ in $N_{1}$ (from Appendix A), add four new vertices inside each face as shown at left in Figure 3 to obtain face-simple $\widetilde{\Theta}_{4}^{\prime}$ in $N_{1}$, and then add a quadrangular crosscap to give $\widetilde{\Theta}_{4}^{\prime \prime}$ in $N_{2}$. Then $4=\chi\left(\widetilde{\Theta}_{4}\right) \leq \chi\left(\widetilde{\Theta}_{4}^{\prime}\right) \leq \chi\left(\widetilde{\Theta}_{4}^{\prime \prime}\right) \leq c\left(N_{1}\right)=c\left(N_{2}\right)=4$, so take $\widetilde{\Theta}_{4}^{\prime}$ and $\widetilde{\Theta}_{4}^{\prime \prime}$ for $N_{1}$ and $N_{2}$, respectively. For $N_{3}$ add a quadrangular crosscap to $\Theta_{5}$ (from Appendix A). If $\Sigma=N_{4}$ or $N_{5}$ use $\Omega_{6, \Sigma}$.

The face-coloring version (b) follows from the vertex coloring-version (a) by taking duals, after applying Lemma 5.2 if an embedding is noncellular. All of the vertex-coloring sharpness examples are quadrangular (implying closed-2-cell), face-simple and simple, so their duals provide face-coloring sharpness examples that are 4-regular, closed-2-cell, simple and face-simple.

We cannot extend the Even Map Color Theorem to embeddings with all face degrees at least 4, because there is no counterpart to Observation 5.3 for such embeddings. They may realize the Heawood bound of the original Map Color Theorem. Suppose $\Phi$ is a sharpness example (such as a triangular embedding of some $K_{n}$ ) for the original Map Color Theorem in a surface $\Sigma \neq S_{0}, N_{2}$, so 
that $\chi(\Phi)=H(\Sigma)$. If we add new vertices inside each triangular face as shown at right in Figure 3, we obtain an embedding $\Phi^{\prime}$ in $\Sigma$ with $\delta^{*}\left(\Phi^{\prime}\right) \geq 4$ and $\chi\left(\Phi^{\prime}\right)=H(\Sigma)>H_{\text {even }}(\Sigma)$.

\section{Embeddings from graphical surfaces and voltage graphs}

In this section, we use graphical surfaces and voltage graphs to construct both orientable and nonorientable quadrangular embeddings of certain graphs of the form $G\left[K_{4}\right]$, proving Theorem 1.5.

Theorem 6.1. Let $G$ be a connected simple graph with a perfect matching. Then $G\left[K_{4}\right]$ has a face-simple orientable quadrangular embedding.

Proof. Let $G$ have perfect matching $M$, and let $S(G)$ be the graphical surface derived from $G$.

First, construct a quadrangular embedding $\Theta$ of $H=G\left[\overline{K_{2}}\right]$ in $S(G)$ as in Lemma 3.6. For each vertex $v \in V(G)$ there are two vertices $v_{\mathrm{N}}, v_{\mathrm{S}} \in V(H)$. For each $u v \in E(G)$, there is a tube $T_{u v}$ in $S(G)$, along which run the edges $u_{\mathrm{N}} v_{\mathrm{N}}, u_{\mathrm{N}} v_{\mathrm{S}}, u_{\mathrm{S}} v_{\mathrm{S}}$ and $u_{\mathrm{S}} v_{\mathrm{N}}$ of $H$. Each edge $u_{P} v_{Q}$ of $H$ belongs to two quadrilaterals of the form $\left(u_{\mathrm{N}} v_{Q} u_{S} t_{X}\right)$ and $\left(u_{P} v_{\mathrm{N}} w_{Y} v_{\mathrm{S}}\right)$ where $t u, v w \in E(G)$ and $P, Q, X, Y \in\{\mathrm{N}, \mathrm{S}\}$.

Modify the embedding $\Theta$ by splitting each edge into a digon (2-cycle) bounding a face. Let $\Psi$ be the new embedding, with underlying graph $J$. The other faces of $\Psi$ are quadrilaterals, in one-to-one correspondence with the quadrilaterals of $\Theta$. We now assign voltages from the group $\mathbb{Z}_{2}$; since all elements of $\mathbb{Z}_{2}$ are self-inverse, the designation of plus directions for edges does not matter. Choose a voltage assignment $\alpha: E(J) \rightarrow \mathbb{Z}_{2}$ so that the voltages of the edges of $J$ around each tube $T_{u v}$ alternate between 0 and 1 . Then each digon of $\Psi$ has one edge of voltage 0 and one edge of voltage 1 . Each quadrilateral of $\Psi$, which uses edges from two tubes $T_{u v}$ and $T_{v w}$, has an edge of voltage 0 and an edge of voltage 1 on $T_{u v}$, and similarly for $T_{v w}$. Therefore, every digon has total voltage 1 and every quadrilateral has total voltage 0 in $\langle J, \alpha\rangle$. Thus, the derived embedding $\Psi^{\alpha}$, with underlying graph $J^{\alpha}=H\left[\overline{K_{2}}\right]=G\left[\overline{K_{2}}\right]\left[\overline{K_{2}}\right]=G\left[\overline{K_{4}}\right]$, is an orientable quadrangulation.

We could also have obtained a quadrangular embedding of $G\left[\overline{K_{4}}\right]=G\left[\overline{K_{2}}\right]\left[\overline{K_{2}}\right]$ directly from Lemma 3.6, but that would not have had the special structure which we now exploit to obtain an embedding of $G\left[K_{4}\right]$. We work with the vertices of $G$ in pairs specified by the perfect matching $M$.

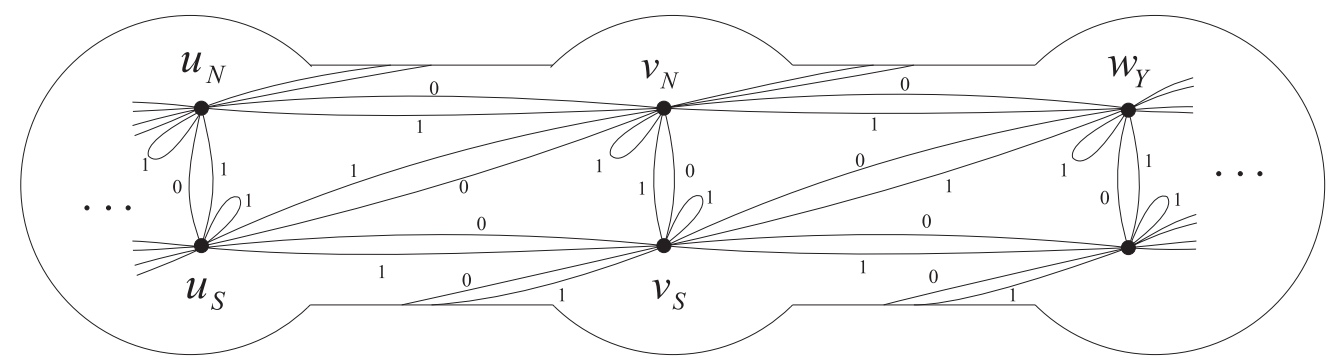

Figure 4: Voltage graph $\left\langle J_{1}, \alpha_{1}\right\rangle$ generated from graphical surface of $G\left[\overline{K_{2}}\right]$.

For each $u v \in M$, let $e$ be one of the four edges of $H$ on the tube $T_{u v}$, between $\left\{u_{\mathrm{N}}, u_{\mathrm{S}}\right\}$ and $\left\{v_{\mathrm{N}}, v_{\mathrm{S}}\right\}$. We choose $e=u_{\mathrm{S}} v_{\mathrm{N}}$, as this makes it easier to illustrate what is happening (see 
Figure 4). In $\Theta, e=u_{\mathrm{S}} v_{\mathrm{N}}$ belongs to two quadrilaterals $Q_{u}=\left(t_{X} u_{\mathrm{N}} v_{\mathrm{N}} u_{\mathrm{S}}\right)$ and $Q_{v}=\left(u_{\mathrm{S}} v_{\mathrm{N}} w_{Y} v_{\mathrm{S}}\right)$ where $t u, v w \in E(G)$ and $X, Y \in\{\mathrm{N}, \mathrm{S}\}$. Let the two edges of the digon in $J$ corresponding to $e$ be $e_{1}$ and $e_{2}$, where $e_{1}$ belongs to the quadrilateral $Q_{u}^{\prime}$ of $\Psi$ corresponding to $Q_{u}$ and $e_{2}$ belongs to the quadrilateral $Q_{v}^{\prime}$ of $\Psi$ corresponding to $Q_{v}$. Add a digon of two edges $d_{1}$ and $d_{2}$ in $Q_{u}^{\prime}$ between $u_{\mathrm{N}}$ and $u_{\mathrm{S}}$, and a digon of two edges $d_{3}$ and $d_{4}$ in $Q_{v}^{\prime}$ between $v_{\mathrm{N}}$ and $v_{\mathrm{S}}$, so we have four triangles $T_{1}(u)=\left(t_{X} u_{\mathrm{N}} u_{\mathrm{S}}\right)$ using $d_{1}, T_{2}(u)=\left(u_{\mathrm{N}} v_{\mathrm{N}} u_{\mathrm{S}}\right)$ using $d_{2}, T_{1}(v)=\left(u_{\mathrm{S}} v_{\mathrm{N}} v_{\mathrm{S}}\right)$ using $d_{3}$ and $T_{2}(v)=\left(v_{\mathrm{S}} v_{\mathrm{N}} w_{Y}\right)$ using $d_{4}$. Assign voltage 1 to $d_{2}$ and $d_{3}$, and 0 to $d_{1}$ and $d_{4}$. Insert a loop with voltage 1 at each of $u_{\mathrm{N}}, u_{\mathrm{S}}, v_{\mathrm{N}}$ and $v_{\mathrm{S}}$ and put these loops in the four different triangles $T_{1}(u), T_{2}(u), T_{1}(v)$ and $T_{2}(v)$, respectively.

Everything up to this point could have been done using independently chosen quadrilaterals $Q_{u}^{\prime}$ containing $u_{\mathrm{N}}$ and $u_{\mathrm{S}}$ and $Q_{v}^{\prime}$ containing $v_{\mathrm{N}}$ and $v_{\mathrm{S}}$. However, the total voltages for the 4 -faces containing the loops at $u_{\mathrm{S}}$ and $v_{\mathrm{N}}$ are currently 1 , so they will not generate quadrilaterals in the derived embedding. To fix this, swap the voltages on $e_{1}$ and $e_{2}$ : this is where we use the pairing of vertices via $M$. Let $\Psi_{1}, J_{1}$ and $\alpha_{1}$ be the final embedding, graph and voltage assignment, as shown in Figure 4.

In $\Psi_{1}$ there are four types of faces. Each 2 -face has total voltage 1 in $\left\langle J_{1}, \alpha_{1}\right\rangle$, and each quadrilateral and 4 -face containing a loop has total voltage 0 . These three types of faces all lift to quadrilaterals in $\Psi_{1}^{\alpha_{1}}$. The final type of face is bounded by a loop of total voltage 1 . This lifts to a face in $\Psi_{1}^{\alpha_{1}}$ bounded by a digon between $\left(u_{X}, 0\right)$ and $\left(u_{X}, 1\right)$, where $u \in V(G)$ and $X \in\{\mathrm{N}, \mathrm{S}\}$. Replacing each such digon in $\Psi_{1}^{\alpha_{1}}$ by a single edge generates the required orientable quadrangular embedding of $G\left[K_{4}\right]$, which is automatically face-simple by Observation 3.4.

As a special case of Theorem 6.1 , by taking $G=K_{2 k}$ for $k \geq 1$ we obtain a proof of Theorem 1.2 if the case where $n \equiv 0(\bmod 8)$. We can also obtain a nonorientable version of Theorem 6.1, which provides a proof of Theorem 1.3 in the case where $n \geq 16$ and $n \equiv 0(\bmod 8)$.

Theorem 6.2. Let $G$ be a connected simple graph with a perfect matching and a cycle. Then $G\left[K_{4}\right]$ has a face-simple nonorientable quadrangular embedding.

Proof. Use Lemma 3.7 instead of Lemma 3.6 in the proof of Theorem 6.1. Replacing one or more tubes by twisted tubes does not affect the argument. Take the orientation-reversing cycle $C=$ $\left(u_{\mathrm{N}} v_{\mathrm{N}} w_{\mathrm{N}} \ldots z_{\mathrm{N}}\right)$ in $H=G\left[\overline{K_{2}}\right]$ from the proof of Lemma 3.7 and replace each edge of $C$ by the edge of voltage 0 in the corresponding digon of $J_{1}$. This gives an orientation-reversing cycle of total voltage 0 in $\left\langle J_{1}, \alpha_{1}\right\rangle$, so the final embedding is nonorientable.

In the nonorientable case we also need to verify that the embedding is face-simple. We can properly 2 -face-color $\Psi_{1}$, coloring the 4 -faces white and the other faces black; this lifts to a proper 2-face-coloring of $\Psi_{1}^{\alpha_{1}}$. In $\Psi_{1}$ each white face shares an edge with four distinct black faces, so this also holds in $\Psi_{1}^{\alpha_{1}}$, and thus $\Psi_{1}^{\alpha_{1}}$ is face-simple. Replacing digons makes each white face share at most one edge with another white face, and the final embedding is still face-simple.

Theorems 6.1 and 6.2 together prove Theorem 1.5. 


\section{Minimal quadrangulations}

In this section we apply our results to determine the order of some minimal quadrangulations.

Hartsfield and Ringel $[14,15]$ showed that an $n$-vertex simple quadrangulation of $\Sigma$ must satisfy $n(n-5) \geq-4 \varepsilon(\Sigma)$. They used this to investigate minimal quadrangulations of surfaces of small genus, and to show that quadrangular embeddings of complete graphs and generalized octahedra $O_{2 k}=K_{k}\left[\overline{K_{2}}\right], k \geq 4$ are minimal. Lawrencenko [21] showed that certain orientable quadrangular embeddings of a graph $G\left[\overline{K_{2}}\right]$, as described in Subsection 3.4, are minimal. The following lemma implies the minimality results of $[14,15,21]$.

Lemma 7.1. Suppose that $L$ is obtained by deleting at most $n-4$ edges from the complete graph $K_{n}, n \geq 5$. Then any quadrangular embedding of $L$ is minimal.

Proof. Let $f(x)=x(x-5) / 2$. Suppose that $x \geq 5$. If $2 \frac{1}{2} \leq x^{\prime} \leq x-1$, then because $f$ is increasing on $\left[2 \frac{1}{2}, \infty\right)$ we have $f(x)-f\left(x^{\prime}\right) \geq f(x)-f(x-1)=x-3$. If $1 \leq x^{\prime} \leq 2 \frac{1}{2}$, then because $2 \frac{1}{2} \leq 5-x^{\prime} \leq x-1$ we have $f(x)-f\left(x^{\prime}\right)=f(x)-f\left(5-x^{\prime}\right) \geq x-3$. Thus, $f(x)-f\left(x^{\prime}\right) \geq x-3$ whenever $1 \leq x^{\prime} \leq x-1$. If $n$ is a nonnegative integer then $f(n)=\left(\begin{array}{l}n \\ 2\end{array}\right)-2 n$.

Now suppose $L$ has $n$ vertices, $m$ edges, and a quadrangular embedding in $\Sigma$. Since at most $n-4$ edges of $K_{n}$ were deleted, $L$, and hence also $\Sigma$, is connected. If we have another quadrangulation of $\Sigma$ with $n^{\prime} \leq n-1$ vertices and $m^{\prime}$ edges, then, since $m^{\prime}=2 n^{\prime}-2 \varepsilon(\Sigma)$ from Observation 1.1,

$$
\begin{aligned}
m^{\prime}-\left(\begin{array}{c}
n^{\prime} \\
2
\end{array}\right) & =2 n^{\prime}-2 \varepsilon(\Sigma)-\left(\begin{array}{c}
n^{\prime} \\
2
\end{array}\right)=-f\left(n^{\prime}\right)-2 \varepsilon(\Sigma)=-f\left(n^{\prime}\right)+m-2 n \\
& \geq-f\left(n^{\prime}\right)+\left(\begin{array}{l}
n \\
2
\end{array}\right)-(n-4)-2 n=f(n)-f\left(n^{\prime}\right)-(n-4) \geq 1,
\end{aligned}
$$

proving that the other graph is not simple.

Lemma 7.1 is sharp whenever $K_{n-1}$ has a quadrangular embedding $\Phi$ of the appropriate orientability type (as in Theorems 1.2 and 1.3). Adding a new vertex of degree 2 adjacent to two opposite vertices of a face of $\Phi$ yields a quadrangular embedding of a graph obtained from $K_{n}$ by deleting $n-3$ edges, but this is not minimal.

We can now apply Lemma 7.1 to Lemmas 3.6 and 3.7, and to Theorems 6.1 and 6.2. The orientable case of Corollary 7.2 is due to Lawrencenko [21, Theorem 2].

Corollary 7.2. Let $k$ and $p$ be integers with $k \geq 4$ and $0 \leq p \leq k / 4-1$. Suppose $G$ is obtained from $K_{k}$ by deleting $p$ edges. Then $G\left[\overline{K_{2}}\right]$ has both orientable and nonorientable quadrangular embeddings that are minimal. Thus, minimal quadrangulations of the orientable surface of genus $k(k-3) / 2-p+1$ and of the nonorientable surface of genus $k^{2}-3 k-2 p+2$ have order $2 k$.

Proof. Deleting $p$ edges from $K_{k}$ does not create isolated vertices or destroy all cycles. Thus, by Lemmas 3.6 and $3.7, G\left[\overline{K_{2}}\right]$ has orientable and nonorientable quadrangular embeddings. These have order $2 k$, and are minimal by Lemma 7.1 since we get $G\left[\overline{K_{2}}\right]$ by deleting $k+4 p \leq 2 k-4$ edges from $K_{2 k}$. We compute the genera of the surfaces from $m=2 n-2 \varepsilon(\Sigma)$. 
Corollary 7.3. Let $\ell$ and $q$ be integers with $\ell \geq 1$ and $0 \leq q \leq(\ell-1) / 2$. Suppose $G$ is obtained from $K_{2 \ell}$ by deleting $q$ edges. Then $G\left[K_{4}\right]$ has both orientable and nonorientable quadrangular embeddings that are minimal. Thus, minimal quadrangulations of the orientable surface of genus $8 \ell^{2}-5 \ell-4 q+1$ and of the nonorientable surface of genus $16 \ell^{2}-10 \ell-8 q+2$ have order $8 \ell$.

Proof. If $\ell=1$ then $q=0$ and $G\left[K_{4}\right]=K_{8 k}$, so orientable and nonorientable quadrangular embeddings exist by Theorems 1.2 and 1.3. If $\ell \geq 2$ then the $q$ edges deleted from $K_{2 \ell}$ are incident with at most $\ell-1$ vertices, so $G$ has $K_{2 \ell}-E\left(K_{\ell-1}\right)$ as a subgraph, and hence has a perfect matching and a cycle. Thus, by Theorems 6.1 and $6.2, G\left[K_{4}\right]$ has the required embeddings.

For all $\ell$ these embeddings have order $8 \ell$, and are minimal by Lemma 7.1 since we get $G\left[K_{4}\right]$ by deleting $16 q<8 \ell-4$ edges from $K_{8 \ell}$. We compute the genera of the surfaces from $m=$ $2 n-2 \varepsilon(\Sigma)$.

The simple quadrangulations described in Corollaries 4.5 and 4.10 are also minimal. The simple quadrangulations with $\ell=n+1$ vertices are embeddings of $K_{\ell}$ with $\ell-4, \ell-5$ or $\ell-6$ edges deleted, and so are minimal by Lemma 7.1.

Corollary 7.4. If $n \equiv 2$ or $3(\bmod 4), n \geq 6$ and $k=2+\lceil n(n-5) / 4\rceil$, then a minimal quadrangulation of $N_{k}$ has $n+1$ vertices. If $n \equiv 1,2,3,4,6$ or $7(\bmod 8), n \geq 7$ and $h=1+\lceil n(n-5) / 8\rceil$, then a minimal quadrangulation of $S_{h}$ has $n+1$ vertices.

There is some overlap here between the conclusions about the order of minimal quadrangulations. The case of Corollary 7.3 with $\ell / 4 \leq q \leq(\ell-1) / 2$ is also covered by Corollary 7.2 with $k=4 \ell$ and $p=4 q-\ell$. Some (but not all) cases of Corollary 7.4 are also covered by Corollary 7.2.

\section{Conclusion}

We give some final remarks.

(1) Hartsfield and Ringel $[14,15]$ defined quadrangulations more strictly than we do: they insisted that two distinct faces share at most one edge and at most three vertices. For an embedding of a simple graph, this is equivalent to being face-simple. The reason for this restriction is unclear. Perhaps they wished to make the embedding "polyhedral". However, an embedding is now usually considered polyhedral if it is a 3-representative (every noncontractible simple closed curve in the surface intersects the graph in at least three points) embedding of a 3-connected graph. A quadrangular embedding of $K_{n}$ is never polyhedral in this sense: given a face (uvwx), the edge $u w$ is part of the boundary of some other face, and using these two faces we can find a simple closed curve intersecting the graph at just $u$ and $w$, which must be noncontractible. In any case, all our embeddings, with a few small exceptions, are face-simple and so satisfy Hartsfield and Ringel's definition.

(2) It may be possible to carry out the graphical surface/voltage graph construction from the proof of Theorem 6.1 with non-perfect matchings $M$ of $G$ as well as with perfect matchings, to give orientable and nonorientable quadrangular embeddings of some graphs $L$ with $G\left[\overline{K_{4}}\right] \subseteq L \subseteq G\left[K_{4}\right]$. This could provide some further examples of minimal quadrangulations. 
(3) Our constructions have a lot of flexibility, particularly the constructions from Subsection 3.4 and Section 6 . The graphical surface embeddings of $G\left[\overline{K_{2}}\right]$ in $S(G)$ (with twisted tubes allowed) require a cyclic order of tubes around the equator of each sphere, and a designation of which tubes are to be twisted. (This corresponds to choosing an arbitrary embedding of $G$, described by a rotation system with edge signatures.) There are two ways to run the edges along each tube. For Theorem 6.1 or 6.2 we may choose an arbitrary perfect matching $M$ of $G$, and for each edge $u v$ of $M$ we may choose one of four possible edges along the corresponding tube to determine $Q_{u}$ and $Q_{v}$. We also have two ways to assign the voltages for the digons of $J$ running along each tube.

It therefore seems natural to ask whether our techniques can be used to provide useful lower bounds on the number of nonisomorphic quadrangular embeddings of $K_{n}$.

\section{A Appendix: Small cases}

In this appendix we provide the embeddings for the bases of the inductive proofs in Section 4 .

\section{A.1 Nonorientable embeddings}

At left in Figure 5 is a face-simple quadrangular embedding $\widetilde{\Psi}_{6}^{-}$of $K_{6}-e(e=01)$ in $N_{3}$, which is used for constructing embeddings related to $K_{5}$. This is shown as a polygon with labeled vertices, indicating how edges are to be identified around the boundary. Nonorientability follows from the existence of edges used twice in the same direction around the outer boundary.

We give nonorientable embeddings $\widetilde{\Theta}_{n}$ for $n \in\{4,6,7,8\}$ in which all faces are $C_{4}$-faces except for possibly one $C_{6}$-face. They are all closed-2-cell and all except $\widetilde{\Theta}_{4}$ are face-simple. The embedding $\widetilde{\Theta}_{4}$ of $K_{4}$ is obtained by taking each of the three hamilton 4-cycles in $K_{4}$ as a face boundary. The

embedding $\widetilde{\Theta}_{6}$ of $K_{6}$ with six $C_{4}$-faces and one $C_{6}$-face is generated by the voltage graph shown at center in Figure 5. The loop of voltage 3 generates digons, which are replaced by single edges. A polygon representation of $\widetilde{\Theta}_{6}$ is also given at right in Figure 5. The embeddings $\widetilde{\Theta}_{7}$ of $K_{7}$ and $\widetilde{\Theta}_{8}$ of $K_{8}$ are shown at left and right, respectively, in Figure 6.

\section{A.2 Orientable embeddings}

Below are orientable embeddings in which all faces are $C_{4}$-faces except for at most two specified faces. These embeddings are represented as rotation systems using vertices $0,1,2, \ldots, 9, \mathrm{a}, \mathrm{b}, \mathrm{c}, \mathrm{d}$. All embeddings are even-faced except for $\Theta_{6}$. All are face-simple and closed-2-cell except $\Theta_{4}$. 

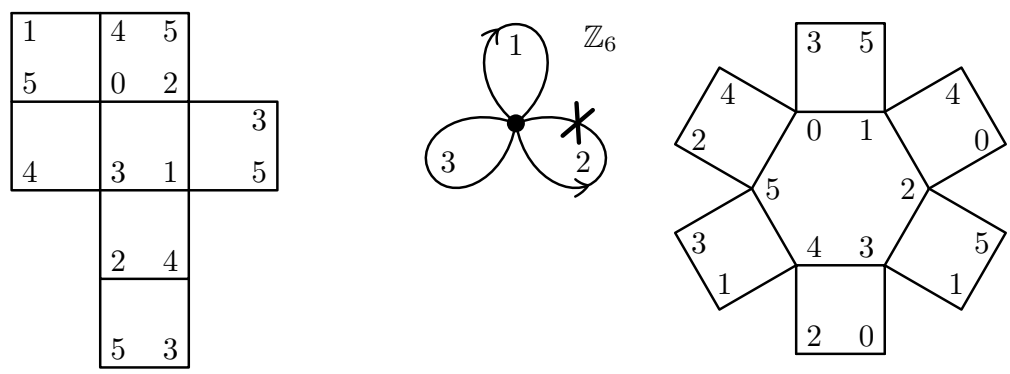

Figure 5: Nonorientable embeddings $\widetilde{\Psi}_{6}^{-}$(left) and $\widetilde{\Theta}_{6}$ (center and right).
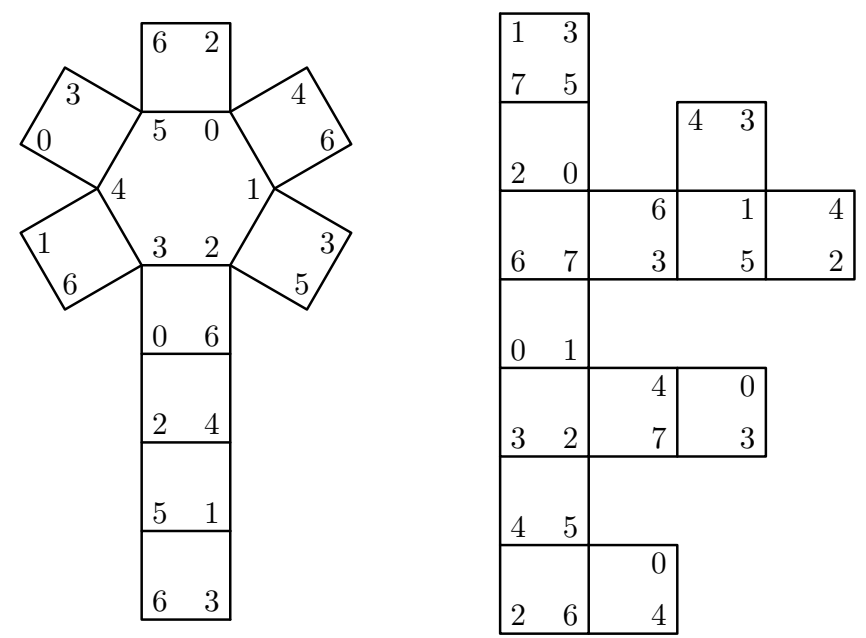

Figure 6: Nonorientable embeddings $\widetilde{\Theta}_{7}$ (left) and $\widetilde{\Theta}_{8}$ (right).

\begin{tabular}{|c|c|}
\hline$\Theta_{4}$ of $K_{4}$ & $\Theta_{5}$ of $K_{5}$ : \\
\hline with 8-face & 0.1423 \\
\hline$(01231032):$ & 1. 0432 \\
\hline 0. 132 & 2. 0341 \\
\hline 1. 023 & 3. 0124 \\
\hline 2. 013 & 4. 0213 \\
\hline 3. 021 & \\
\hline
\end{tabular}

$$
\begin{aligned}
& \Theta_{6} \text { of } K_{6} \\
& \quad \text { with } C_{5} \text {-faces } \\
& \quad(01234),(03142) \text { : }
\end{aligned}
$$

0. 12354

1. 02345

2. 05134

3. 01524

4. 01253

5. 04321
$\Theta_{7}$ of $K_{7}$ with $C_{6}$-face (012345):

0. 143265

1. 025346

2. $06 \begin{array}{llll}6 & 1 & 3 & 5\end{array}$

3. 012456

4. 062351

5. 032164

6. 041523 

$\Theta_{8}$ of $K_{8}$ :
0. 1743526
1. 0725643
2. 0734615
3. 0716524
4. 0612537
5. 0431627
6. 0521347
7. 0342516

$$
\begin{aligned}
& \Theta_{9} \text { of } K_{9} \text { with } C_{8} \text {-face } \\
& \text { (01234567): }
\end{aligned}
$$

0. 14628537

1. 02643758

2. 08576413

3. 08246175

4. 08623571

5. 07321468

6. 01457328

7. 05823416

8. 03541267

$\Theta_{11}$ of $K_{11}$ with $C_{10}$-face (0123456789):

0. 1573 a 64829

1. $027964583 \mathrm{a}$

2. 0 a 13569847

3. 0 a 72419865

4. 0 a 35268197

5. 0 a 32746189

6. $091234857 \mathrm{a}$

7. $092536841 \mathrm{a}$

8. $079162543 \mathrm{a}$

9. 0752146 a 38

a. 0514637289
$\Theta_{12}$ of $K_{12}$ with $C_{8}$-face (01234567):
0. 1 a 29384 b 657
1. 02 b 658473 a 9
2. 0 b 75138469 a
3. 0 b $78596241 \mathrm{a}$
4. 0 b 1 a 3582967
5. 0 b $28734691 \mathrm{a}$
6. 0 a 19428357 b
7. 0 a 1 b 4953826
8. 0 a $19264357 \mathrm{~b}$
9. 0 a 12485376 b
a. $0921483756 \mathrm{~b}$
b. 0748391562 a

$\Theta_{10}$ of $K_{10}$ with $C_{10}$-face (0123456789):

0. 156473829

1. 024367589

2. 098761354

3. 091824576

4. 092357816

5. 094671328

6. 083574219

7. 068531249

8. 065342179

9. 046375128

$\Theta_{14}$ of $K_{14}$ with $C_{6}$-face (012345):

0. 17 a 4 b 3 c 2 d 8695

1. 0267 a 3 b 9485 c d

2. 0 d c 139 b a 48576

3. 0 d 24691 c 578 a b

4. 0 d 687935 a 2 b 1 c

5. 0 d 7 a 831 c 2 b 964

6. 0 d 2 a 539487 b $1 \mathrm{c}$

7. 0 d 691 b 4385 a 2 c

8. 0 c 1 b 2 a 753496 d

9. 0 c 1 a 7864 b 352 d

a. 0 c 19 b $2358467 \mathrm{~d}$

b. 0 c 32584 a 1976 d

c. 0 b 496213 a 758 d

d. 0684 a 593 b 712 c

\section{B Appendix: Analysis of $K_{6}$ in $S_{2}$}

In this appendix we prove Proposition 4.8, and show that $K_{6}$ has no even-faced general embedding in $S_{2}$.

In an embedding the faces around a vertex must form a proper rotation, a cyclic sequence closing up so that the vertex has a neighborhood homeomorphic to an open disk. If a potential set of faces incident with a vertex $v$ close up in a cyclic sequence without including all edges incident with $v$, we say the rotation at $v$ is improperly closed.

Lemma B.1. $K_{6}$ has no orientable embedding in which every face is a $C_{4}$-face except for one $C_{6}$-face.

Proof. Assume that such an embedding exists. Since the embedding is orientable we may assign consistent orientations to all the faces, and describe each face using a cyclic list of vertices following the orientation (it is not equivalent to its reverse).

We will label the vertices of $K_{6}$ by elements of $\mathbb{Z}_{6}=\{0,1,2,3,4,5\}$. Each edge has an obviously defined length of 1,2 or 3 depending on $j-i$. Each arc (directed edge) from $i$ to $j$ has length $j-i \in \mathbb{Z}_{6}$ 
which we will write as an element of the set $\{-2,-1,1,2,3\}$ (where $-2=4,-1=5$ ). Each arc is used exactly once by our embedding. Without loss of generality we may label the vertices so that the $C_{6}$-face is $Z=(054321)$, using all the arcs of length -1 . Therefore the remaining $6 C_{4}$-faces use 6 arcs of each length $1,2,-2$ and 3 . Since no $C_{4}$ in $K_{6}$ can use more than two arcs of length \pm 2 , and altogether they use 12 arcs of length \pm 2 , each of the $6 C_{4}$ faces must use exactly two arcs of length \pm 2 .

Therefore the cyclic pattern of lengths in each $C_{4}$-face (following the $\operatorname{arcs}$ in their positive direction) must be one of 6 possibilities: $A=(1,1,2,2), B=(1,2,1,2), C=(1,-2,-2,3)$, $D=(1,3,-2,-2), E=(1,-2,3,-2)$ or $F=(2,3,-2,3)$. For each pattern $P$ let $P_{i}$ be the potential face starting at vertex $i$ and following pattern $P$; for example, $C_{3}=(3420)(3+1=4$, $4-2=2,2-2=0,0+3=3)$.

Any face $A_{i}$ together with $Z$ improperly closes the rotation at $i+1$, so there are no faces of pattern $A$. Let $n_{F}$ be the number of faces of pattern $F$ and $n_{C D E}$ the number of faces of pattern $C, D$ or $E$. Counting the arcs of length -2 we have $2 n_{C D E}+n_{F}=6$. Counting the arcs of length 3 we have $n_{C D E}+2 n_{F}=6$. Therefore $n_{C D E}=n_{F}=2$.

Consider the two faces of pattern $F$. They must share an edge of length 3 , which we may assume is 03. The faces of pattern $F$ using this edge are $F_{3}$ and $F_{4}$, which use arc 03 , and $F_{0}$ and $F_{1}$, which use arc 30. We must have one face that uses arc 03 and one that uses arc 30. Moreover, we cannot have $F_{3}$ and $F_{0}$ because they are reverses of each other, giving improper rotations at all of their vertices if they occur together in an embedding. Similarly, we cannot have $F_{4}$ and $F_{1}$, because they are reverses of each other. So we must have $F_{3}$ and $F_{1}$, or $F_{4}$ and $F_{0}$. Without loss of generality we assume we have $F_{4}$ and $F_{0}$; if we have $F_{3}$ and $F_{1}$ we just add 3 to all the vertex labels and they become $F_{0}$ and $F_{4}$.

Now consider the arc 01, which must belong to some face. The possible faces are $B_{0}=B_{3}, C_{0}$, $D_{0}$ or $E_{0}$. If $B_{0}$ is a face then the arc 45 is used by both $B_{0}$ and $F_{4}$. If $C_{0}$ is a face then the $\operatorname{arcs}$ 53 and 30 are used by both $C_{0}$ and $F_{0}$, a contradiction. If $D_{0}$ is a face then the arc 14 is used by both $D_{0}$ and $F_{4}$, a contradiction. If $E_{0}$ is a face then $E_{0}$ and $F_{0}$ improperly close up the rotation at vertex 2 , which is a contradiction.

Hence all situations lead to a contradiction, so, as claimed, there is no such embedding of $K_{6}$.

Lemma B.2. Every cellular orientable embedding of $K_{6}$ in which some vertex is incident with five $C_{4}$-faces must have five $C_{4}$-faces and two $C_{5}$-faces.

Proof. Label the vertices of $K_{6}$ by $\infty$ and the elements of $\mathbb{Z}_{5}=\{0,1,2,3,4\}$. Without loss of generality we may suppose that $\infty$ has clockwise rotation $(0,1,2,3,4)$, and that for $i \in \mathbb{Z}_{5}$ there is a 4-cycle face $\left(\infty, i, a_{i+3}, i+1\right)$ (writing faces also in clockwise order; this labelling makes $a_{i}$ 'opposite' to $i$ in the face neighborhood around $\infty)$. For each $i \in \mathbb{Z}_{5}$ we must have $a_{i} \notin\{\infty, i-3, i-2\}$, so $a_{i} \in\{i-1, i, i+1\}$ for each $i$; let the rotation around $i$ be $\left(\infty, a_{i+2}, b_{i 1}, b_{i 2}, a_{i+3}\right)$. Since the same vertex cannot occur twice in the rotation around $i-2, a_{i} \neq a_{i+1}$ for each $i$.

Suppose first that $a_{i}=a_{j}$ for some $i \neq j$. The only way this can happen is if $j=i \pm 2$ and $a_{i}$ is the number between $i$ and $j$. Without loss of generality suppose that $a_{4}=a_{1}=0$. Then the rotation around 0 contains the sequence 4,3 since $a_{1}=0$ and the sequence 2,1 since $a_{4}=0$. Since 
$a_{2} \neq 4$, the rotation around 0 must be $\left(\infty, a_{2}=2,1,4, a_{3}=3\right)$. Since $a_{3}=3$, the rotation around 3 contains the sequence 1,0 ; but we already know that the rotation around 3 contains the sequence $b_{32}, a_{1}=0$ and hence $b_{32}=1$. This means that $a_{0} \neq 1$. By similar reasoning, $b_{21}=4$ and hence $a_{0} \neq 4$. Also $a_{0} \neq a_{4}=0$. But now there are no possible values for $a_{0}$, which is a contradiction. So we know that $a_{i} \neq a_{j}$ when $i \neq j$. Hence each $j \in \mathbb{Z}_{5}$ occurs exactly once as some $a_{i}$.

Suppose that $a_{0}=1$. The rotation around $a_{0}=1$ contains the sequence 3,2 , so we have $(3,2)=\left(a_{3}, b_{11}\right),\left(b_{11}, b_{12}\right)$ or $\left(b_{12}, a_{4}\right)$. We cannot have $a_{4}=2$ so $(3,2)$ cannot be $\left(b_{12}, a_{4}\right)$. If $(3,2)=\left(a_{3}, b_{11}\right)$ then both $1=a_{0}$ and $3=a_{3}$ occur as vertices $a_{i}$, so we must have $a_{2}=2$. Then the rotation around $a_{2}=2$ contains the sequence 0,4 . Now $a_{4} \neq a_{3}=3$ so $a_{4} \in\{0,4\}$ and the rotation around 2 contains the sequence $\infty, a_{4}$, so we cannot have $a_{4}=4$ and we must have $a_{4}=0$. But now 1,2,0 have all been used as vertices $a_{i}$, so there is no valid value for $a_{1}$, a contradiction. Hence we must have $(3,2)=\left(b_{11}, b_{12}\right)$. But then $a_{3}$ cannot be equal to either 3 or 2 , so $a_{3}=4$.

Generalizing the above, we have shown that $a_{i}=i+1$ implies that $a_{i+3}=i+4$, and $b_{i+1,1}=i+3$, $b_{i+1,2}=i+2$. Repeating this reasoning determines the rotation around every vertex $i$ as being $(\infty, i+3, i+2, i+1, i-1)$. This gives an embedding of $K_{6}$ with five $C_{4}$-faces and two $C_{5}$-faces (03142) and (01234), in clockwise order.

The case where $a_{i}=i-1$ for some $i$ is symmetric. So we need only deal with the case where $a_{i}=i$ for all $i$. However, this is impossible: for example, it leads to the rotation around vertex 0 containing $a_{3}, \infty=3, \infty$ but also the rotation around $a_{0}=0$ containing 3,2 .

Thus, the only possible situations lead to the embedding specified.

Proof of Proposition 4.8. Assume there is a general embedding $\Phi$ of $K_{6}$ in $S_{2}$. As noted in Section 4, $\Phi$ must be cellular with six $C_{4}$-faces and one 6 -face, or five $C_{4}$-faces and two $C_{5}$-faces.

Suppose there is a 6 -face. By Lemma B.1 the 6 -face is not a $C_{6}$-face. So the 6 -face has fewer than 6 distinct vertices, and thus there is some vertex all of whose adjacent faces are $C_{4}$-faces. But then, by Lemma B.2, $\Phi$ does not have a 6 -face, a contradiction.

Therefore the embedding has five $C_{4}$-faces and two $C_{5}$-faces. The embedding $\Theta_{6}$ in Appendix A, or the embedding found in the proof of Lemma B.2, shows that such an embedding exists.

\section{Acknowledgements}

The authors thank Joan Hutchinson for information regarding [13].

Wenzhong Liu was partially supported by Fundamental Research Funds for the Central Universities (NZ2015106) and NSFC grant (11471106). M. N. Ellingham was partially supported by National Security Agency grant H98230-13-1-0233 and Simons Foundation award no. 429625. Dong Ye was partially supported by Simons Foundation award no. 359516. Xiaoya Zha was partially supported by National Security Agency grant H98230-13-1-0216. 


\section{References}

[1] A. Altshuler and U. Brehm, Neighborly maps with few vertices, Discrete Comput. Geom. 8 (1992) 93-104.

[2] C.P. Bonnington, M.J. Grannell, T.S. Griggs and J. Širáň, Exponential families of nonisomorphic triangulations of complete graphs, J. Combin. Theory Ser. B 78 (2000) 169-184.

[3] A. Bouchet, Orientable and nonorientable genus of the complete bipartite graph, J. Combin. Theory Ser. B 24 (1978) 24-33.

[4] Beifang Chen, Serge Lawrencenko and Hui Yang, Determination of the 4-genus of a complete graph, preprint, 1998; posted at https://www.researchgate.net/publication/ 306255986, 2016.

[5] D.L. Craft, Surgical techniques for constructing minimal orientable imbeddings of joins and compositions of graphs, Ph.D. thesis, Western Michigan University, 1991.

[6] David Craft, Quadrilateral embeddings of composition graphs, 1995, problem posted by Dan Archdeacon at http://www.cems.uvm.edu/TopologicalGraphTheoryProblems/ composit.htm, downloaded 2 August 2018.

[7] D.L. Craft, On the genus of joins and compositions of graphs, Discrete Math. 178 (1998) $25-50$.

[8] M.J. Grannell, T.S. Griggs and J. Širán̆, A lower bound for the number of triangular embeddings of some complete graphs and complete regular tripartite graphs, J. Combin. Theory Ser. B 98 (2008) 637-650.

[9] M.J. Grannell and M. Knor, A lower bound for the number of orientable triangular embeddings of some complete graphs, J. Combin. Theory Ser. B 100 (2010) 216-225.

[10] M.J. Grannell and T.A. McCourt, Doubly even orientable closed 2-cell embeddings of the complete graph, Electron. J. Combin. 21 (2014) P1.22, 17pp.

[11] J. L. Gross and T. W. Tucker, Topological Graph Theory, Dover, Mineola, New York, 2001.

[12] Nora Hartsfield, Nonorientable quadrangular embeddings of complete multipartite graphs, Proc. 25th Southeastern Internat. Conf. on Combinatorics, Graph Theory and Computing (Boca Raton, FL, 1994), Congr. Numer. 103 (1994) 161-171.

[13] Nora Hartsfield, The quadrangular genus of complete graphs, preprint, 1994.

[14] N. Hartsfield and G. Ringel, Minimal quadrangulations of orientable surfaces, J. Combin. Theory Ser. B 46 (1989) 84-95.

[15] N. Hartsfield and G. Ringel, Minimal quadrangulations of nonorientable surfaces, J. Combin. Theory Ser. A 50 (1989) 186-195.

[16] Joan P. Hutchinson, On coloring maps made from Eulerian graphs, Proc. Fifth British Combinatorial Conf. (Univ. Aberdeen, Aberdeen, 1975), Congr. Numer. 15 (1976) 343-354.

[17] Joan P. Hutchinson, Three-coloring graphs embedded on surfaces with all faces even-sided, J. Combin. Theory Ser. B 65 (1995) 139-155. 
[18] K. Kawarabayashi, C. Stephens and X. Zha, Orientable and nonorientable genera of some complete tripartite graphs, SIAM J. Discrete Math. 18 (2004) 479-487.

[19] V.P. Korzhik, Generating nonisomorphic quadrangular embeddings of a complete graph, $J$. Graph Theory 74 (2013) 133-142.

[20] V.P. Korzhik and H.-J. Voss, On the number of nonisomorphic orientable regular embeddings of complete graphs, J. Combin. Theory Ser. B 81 (1) (2001) 58-76.

[21] Serge Lawrencenko, Realizing the chromatic numbers and orders of spinal quadrangulations of surfaces, J. Combin. Math. Combin. Comput. 87 (2013) 303-308.

[22] Serge Lawrencenko, Beifang Chen and Hui Yang, Determination of the 4-genus of a complete graph (with an appendix), arXiv:1803.03855v1, 2018.

[23] Serge Lawrencenko, Beifang Chen, Hui Yang and Nora Hartsfield, The orientable 4-Genus Formula for the complete graph, in preparation.

[24] S. Lawrencenko, S. Negami and A.T. White, Three nonisomorphic triangulations of an orientable surface with the same complete graph, Discrete Math. 135 (1994) 367-369.

[25] Wenzhong Liu, M.N. Ellingham, Dong Ye and Xiaoya Zha, Quadrangular embeddings of complete graphs, arXiv:1606.00948v1, 2016.

[26] Wenzhong Liu, Serge Lawrencenko, Beifang Chen, M.N. Ellingham, Nora Hartsfield, Hui Yang, Dong Ye and Xiaoya Zha, Quadrangular embeddings of complete graphs and the Even Map Color Theorem, J. Combin. Theory Ser. B 139 (2019) 1-26.

[27] Z. Magajna, B. Mohar and T. Pisanski, Minimal ordered triangulations of surfaces, J. Graph Theory 10 (1986) 451-460.

[28] B. Mohar, T.D. Parsons, and T. Pisanski, The genus of nearly complete bipartite graphs, Ars Combin. 20 (1985) 173-183.

[29] G. Ringel, Das Geschlecht des vollständigen paaren Graphen, Abh. Math. Sem. Univ. Hamburg. 28 (1965) 139-150.

[30] G. Ringel, Der vollständige paare Graph auf nichtorientierbaren Flächen, J. Reine Angew. Math. 220 (1965) 88-93.

[31] G. Ringel, Map Color Theorem, Springer, Berlin, 1974.

[32] Yusuke Suzuki, Triangulations on closed surfaces which quadrangulate other surfaces II, Discrete Math. 303 (2005) 235-243.

[33] Arthur T. White, On the genus of the composition of two graphs, Pacific J. Math. 41 (1972) 275-279.

[34] J.W.T. Youngs, The mystery of the Heawood conjecture, in 'Graph Theory and its Applications,' Proc. Advanced Sem., Math. Research Center, Univ. of Wisconsin, Madison, Wis., 1969, Academic Press, New York, 1970, pp. 17-50. 